\title{
$\beta$ Cephei stars in the ASAS-3 data
}

\section{103 new $\beta$ Cephei stars and a discussion of low-frequency modes ${ }^{\star}, \star$}

\author{
A. Pigulski ${ }^{1}$ and G. Pojmański ${ }^{2}$ \\ 1 Instytut Astronomiczny Uniwersytetu Wrocławskiego, Kopernika 11, 51-622 Wrocław, Poland \\ e-mail: pigulski@astro.uni.wroc.pl \\ 2 Obserwatorium Astronomiczne Uniwersytetu Warszawskiego, Al. Ujazdowskie 4, 00-478 Warszawa \\ e-mail: gp@astrouw.edu.pl
}

Received 30 August 2007 / Accepted 7 November 2007

\begin{abstract}
Context. The $\beta$ Cephei stars have been studied for over a hundred years. Despite this, many interesting problems related to this class of variable stars remain unsolved. Fortunately, these stars seem to be well-suited to asteroseismology. Hence, the results of seismic analysis of $\beta$ Cephei stars should help us to better understand pulsations and the main sequence evolution of massive stars, particularly the effect of rotation on mode excitation and internal structure. It is therefore extremely important to increase the sample of known $\beta$ Cephei stars and select targets that are useful for asteroseismology.

Aims. We analysed ASAS-3 photometry of bright early-type stars with the goal of finding new $\beta$ Cephei stars. We were particularly interested in $\beta$ Cephei stars that would be good for seismic analysis, i.e., stars that (i) have a large number of excited modes; (ii) show rotationally split modes; (iii) are components of eclipsing binary systems; (iv) have low-frequency modes, that is, are hybrid $\beta$ Cephei/SPB stars.

Methods. Our study was made with a homogeneous sample of over 4100 stars having MK spectral type B5 or earlier. For these stars, the ASAS-3 photometry was analysed by means of a Fourier periodogram.

Results. We have discovered $103 \beta$ Cephei stars, nearly doubling the number of previously known stars of this type. Among these stars, four are components of eclipsing binaries, seven have modes equidistant or nearly equidistant in frequency. In addition, we found five $\beta$ Cephei stars that show low-frequency periodic variations, very likely due to pulsations. We therefore regard them as candidate hybrid $\beta$ Cephei/SPB pulsators. All these stars are potentially very useful for seismic modeling. Moreover, we found $\beta$ Cephei-type pulsations in three late O-type stars and fast period changes in one, HD 168050.
\end{abstract}

Key words. stars: early-type - stars: oscillations - stars: binaries: eclipsing - stars: variables: general - surveys

\section{Introduction}

This is the second paper in the series presenting results of a search for $\beta$ Cephei-type pulsating variables in the $V$-filter photometry in the third phase of the All Sky Automated Survey, ASAS-3 (Pojmański 1997, 2000; Pojmański et al. 2005). In the first paper of the series (Pigulski \& Pojmański 2008, hereafter Paper I), we published the results of an analysis of the ASAS-3 photometry for 41 of about 112 presently known $\beta$ Cepheitype stars, that is, all that had sufficiently good photometry in the ASAS-3 database. The analysis allowed us to detect amplitude changes in three $\beta$ Cephei stars, BW Cru, V836 Cen and V348 Nor, and period changes in KK Vel and V836 Cen. In addition, new modes were found or ambiguities in the frequencies of previously detected modes were removed for some stars.

The published ASAS catalogues, containing photometry of variable star candidates, have already been used to discover $\beta$ Cephei stars. In total, 19 new members of this group have

* Table 2 and Figs. 2-14 are only available in electronic form at http://www. aanda.org

$\star \star$ The $V$ photometry for all 103 stars is available in electronic form at the CDS via anonymous ftp to cdsarc.u-strasbg.fr (130.79.128.5) or via

http://cdsweb.u-strasbg.fr/cgi-bin/qcat?J/A+A/477/917 been found in the ASAS data (Pigulski 2005; Handler 2005). However, owing to the fact that the method of selection of variable stars was biased towards large-amplitude variables, all these 19 stars had relatively large amplitudes. Therefore, it was obvious that once a detailed analysis of the whole ASAS-3 database has been done, many new stars of this type, with smaller amplitudes, will be found. Such an analysis will be carried out in the future, but because the whole ASAS database contains millions of stars and the period(s) alone are not sufficient to classify a given star as a $\beta$ Cephei variable, we decided to proceed in a different way. Using the information in the existing stellar catalogues, we selected stars that are known to be either O-type or early B-type stars and analysed ASAS-3 photometry for these stars only. The selection criteria are explained in Sect. 2. As a result, nearly $300 \beta$ Cephei stars were discovered of which the first part is discussed in this paper. This will allow us to define the properties of the whole class much better and, in view of the increasing interest in the study of these stars by means of asteroseismology, to select appropriate targets for subsequent work. In the present paper we describe the results of the search for $\beta$ Cephei-type stars among objects selected from the five volumes of the Michigan Catalogue for HD stars. The ASAS-3 photometry for stars selected using other catalogues will be discussed in the next paper. 


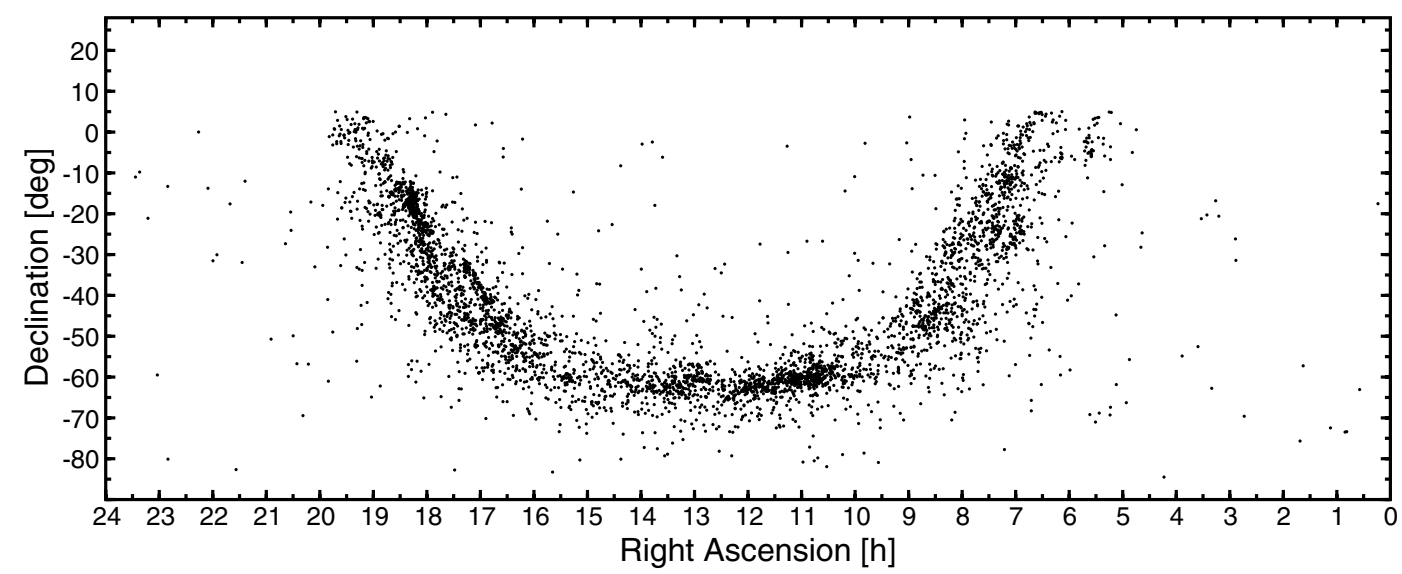

Fig. 1. Location in equatorial coordinates of all stars with $V>7 \mathrm{mag}, \delta<+5^{\circ}$ and of spectral type B5 or earlier, selected from Michigan Catalogues as candidates for $\beta$ Cephei stars.

\section{Selection of early-type stars}

The existing catalogues contain many different types of data that allow selecting early-type stars. The early B and O-type stars can be best selected using either the information on spectral types or the $U B V$ photometry. For this reason, the obvious choice of the source of the information on spectral types was the Michigan Catalogue of Two-Dimensional Spectral Types for the HD stars (Houk \& Cowley 1975; Houk 1978, 1982; Houk \& Smith-Moore 1988; Houk \& Swift 1999). The five volumes of this catalogue contain MK spectral types for 161472 stars south of declination $+5^{\circ}$. The stars are the same as those in The Henry Draper Catalogue (Cannon \& Pickering 1918-1924), i.e., are brighter than $\sim 10$ mag in $V$. This means that all stars fainter than $\sim 7$ mag with HD numbers will have good ASAS-3 photometry because the ASAS survey covers the range of $V$ magnitudes between 7 and $14 \mathrm{mag}$.

While selecting stars from the Michigan Catalogue, we used only two conditions: (i) $V>7$ mag (brighter stars are overexposed in the ASAS $V$-filter frames); (ii) MK spectral type B5 or earlier. In this way, about 4100 stars were selected. Their equatorial coordinates are plotted in Fig. 1. As expected, the stars concentrate along the Galactic plane. For all these stars, the ASAS-3 photometry was extracted, and subsequently analysed by means of Fourier periodograms in the frequency range from 0 to $40 \mathrm{~d}^{-1}$.

A star was regarded as variable if the signal-to-noise $(S / N)$ of the highest peak in the periodogram exceeded 5 .

\section{The results}

In total, $103 \beta$ Cephei stars were found in the sample of about 4100 stars selected using Michigan Catalogue. These stars are listed in Table 1; the frequencies, amplitudes and times of maximum light for the detected modes are presented in Table 2, available online. In addition, periodograms showing the consecutive steps of prewhitening are shown in Figs. 2-14, also available at CDS.

The ASAS-3 photometry we analysed was carried out with a Johnson $V$ filter. The data cover the time interval between the beginning of the project in 2000 and the end of February, 2006. As already shown in Paper I, the ASAS-3 data suffer from daily aliases. Consequently, the frequencies of some modes we provide in Table 2, especially of those with the smallest amplitudes, might be in error by 1 or even 2 cycles per sidereal day. On the other hand, the yearly aliases are relatively low in the ASAS-3 data (see Paper I).

\subsection{Detected modes}

With a detection threshold typical for one-site ground-based data, up to several modes were detected in a single $\beta$ Cephei star. Currently, the largest number of independent modes, fourteen, was observed in $v$ Eridani as a result of a large photometric campaign (Jerzykiewicz et al. 2005). Another $\beta$ Cephei star with large number of detected modes, namely eleven, is 12 (DD) Lacertae (Handler et al. 2006). A histogram showing the number of detected modes in our sample of the 103 new $\beta$ Cephei stars is shown in Fig. 15. In total, 198 independent modes were detected. Up to six modes were found in a single star; there are two such objects, HD 74339 and HD 86214. Some stars with the largest number of detected modes will be discussed in Sect. 3.3. The detection threshold, DT, defined as four times the average amplitude in the Fourier periodogram of the residuals in the range $0-40 \mathrm{~d}^{-1}$, is shown with open circles in Fig. 16 and listed in Table 2. Typically, it amounts to 3-5 mmag. On the other hand, the standard deviation of the residuals, RSD, which can be regarded as the mean accuracy of a single measurement, equals to 10-15 mmag, depending on the magnitude (Fig. 16, see also Table 2). In addition, it can be seen from Fig. 16 that all but several stars have $V$ magnitudes in the range between 8 and 10, i.e., are quite bright. The brightest $\beta$ Cephei-type star we found, at magnitude $V=7.19$, is HD 149100 .

\subsection{Amplitudes and periods}

Among stars listed in the review paper of Stankov \& Handler (2005), the well-known $\beta$ Cephei-type BW Vul is exceptional because of its high amplitude. As we can see from Fig. 17, the stars discovered in the ASAS data by Handler (2005) and Pigulski (2005) fill the gap in amplitudes between BW Vul and the other stars. One star from that sample, HDE 328862, has the amplitude similar to that of BW Vul. For another one, CPD $-62^{\circ} 2707$, a harmonic of the main mode, an indication of non-sinusoidal light curve, was detected. In our list of newly discovered $\beta$ Cephei stars there is another star with an exceptionally large amplitude, HD 173006.

BW Vulpeculae is known for its unusual non-sinusoidal light curve, with a standstill on the raising branch (Fig. 18). It is interesting to note that a standstill was also found in the 
Table 1. New $\beta$ Cephei stars. $N$ is the number of independent modes found in the ASAS-3 photometry, the remaining columns are self-explanatory. References to the MK spectral types in the eighth column are the following: (1) Houk \& Cowley (1975); (2) Houk (1978); (3) Houk (1982); (4) Houk \& Smith-Moore (1988); (5) Houk \& Swift (1999); (6) Hill (1970).

\begin{tabular}{|c|c|c|c|c|c|c|c|c|}
\hline HD & CPD/BD & ASAS name & $N$ & $\begin{array}{c}V \\
{[\mathrm{mag}]}\end{array}$ & $\begin{array}{l}B-V \\
{[\mathrm{mag}]}\end{array}$ & $\begin{array}{l}U-B \\
{[\mathrm{mag}]}\end{array}$ & MK sp. type & Notes \\
\hline 46994 & $-25^{\circ} 1525$ & $063513-2550.4$ & 1 & 7.86 & -0.17 & - & $\mathrm{B} 2 / 3 \mathrm{~V}(4)$ & in CMa OB2 \\
\hline 48553 & $+02^{\circ} 1369$ & $064410+0223.5$ & 1 & 9.03 & - & _ & B2 III (5) & in the CoRoT field \\
\hline 67600 & $-20^{\circ} 3392$ & 080754-2109.0 & 1 & 9.18 & - & - & B3/5 V (4) & \\
\hline 68962 & $-35^{\circ} 2054$ & $081323-3618.7$ & 1 & 7.34 & +0.15 & -0.65 & $\mathrm{~B} 2 / 3 \mathrm{~V}(3)$ & in $\mathrm{BH} 23$ \\
\hline 69016 & $-27^{\circ} 2973$ & $081401-2743.1$ & 1 & 10.22 & - & - & B3 IV (3) & \\
\hline 69824 & $-48^{\circ} 1585$ & $081638-4826.2$ & 1 & 9.10 & - & - & $\mathrm{B} 4 / 6 \mathrm{~V}(2)$ & \\
\hline 73568 & $-44^{\circ} 2824$ & $083719-4512.5$ & 2 & 8.35 & +0.30 & -0.57 & B $2 / 3$ IV (2) & in Vela OB1 \\
\hline 74339 & $-47^{\circ} 2545$ & $084133-4801.5$ & 6 & 9.30 & +0.14 & -0.67 & B2/3 II/III (2) & in IC 2395 \\
\hline 77769 & $-46^{\circ} 3350$ & $090248-4657.9$ & 1 & 9.38 & - & - & B3 IV (2) & in Platais 9 \\
\hline 86085 & $-38^{\circ} 3738$ & 095519-3845.8 & 1 & 8.94 & -0.09 & -0.85 & B1 Vn (6) & halo star \\
\hline 86214 & $-59^{\circ} 1528$ & $095458-5949.8$ & 6 & 9.21 & -0.02 & -0.79 & B2 Ib (1) & \\
\hline 86248 & $-30^{\circ} 3001$ & $095633-3126.5$ & 1 & 9.63 & -0.19 & -0.89 & B2 III (3) & high-velocity star, very distant \\
\hline 87592 & $-58^{\circ} 1860$ & $100409-5922.9$ & 5 & 8.96 & - & - & B1 IV (1) & \\
\hline 88844 & $-60^{\circ} 1765$ & $101302-6110.7$ & 2 & 8.53 & -0.16 & -0.93 & B0.5 III (1) & ALS 1453 \\
\hline 90075 & $-59^{\circ} 2079$ & $102206-6011.2$ & 1 & 8.88 & +0.07 & -0.76 & B1 II (1) & ALS 1517 \\
\hline 90987 & $-57^{\circ} 3308$ & $102849-5746.0$ & 4 & 9.64 & +0.13 & -0.70 & B1/2 III/IVp (1) & ALS 1601, in IC 2581? \\
\hline 91651 & $-59^{\circ} 2214$ & $103330-6007.7$ & 2 & 8.85 & -0.01 & -0.89 & $08 / 9 \mathrm{~V}(1)$ & ALS 1647, in Car OB1 \\
\hline 92291 & $-60^{\circ} 2077$ & $103756-6116.5$ & 5 & 9.07 & -0.07 & -0.86 & B2 Ib/II (1) & ALS 1702 \\
\hline 93113 & $-57^{\circ} 3449$ & $104400-5741.1$ & 1 & 8.71 & +0.03 & -0.76 & B1/2 II/III (1) & ALS 1818 \\
\hline 93341 & $-56^{\circ} 3759$ & $104524-5659.4$ & 1 & 9.64 & +0.02 & -0.74 & B1/2 II/III (1) & ALS 1876 \\
\hline 94065 & $-61^{\circ} 1905$ & $105007-6148.3$ & 5 & 8.95 & -0.05 & -0.83 & B0/1 III-IV (1) & \\
\hline 94345 & $-59^{\circ} 2802$ & $105210-5957.3$ & 3 & 9.00 & +0.06 & -0.77 & B0/1 IV (1) & \\
\hline 94900 & $-59^{\circ} 2859$ & $105611-5955.8$ & 1 & 9.25 & +0.17 & -0.66 & B1/2 III (1) & ALS 2034 \\
\hline 95568 & $-61^{\circ} 2021$ & $110040-6236.9$ & 2 & 9.56 & +0.12 & - & O9/B0 V (1) & ALS 2084 \\
\hline 96882 & $-60^{\circ} 2553$ & $110824-6117.1$ & 1 & 9.03 & -0.03 & -0.86 & B1 II/III (1) & \\
\hline 96901 & $-63^{\circ} 1845$ & $110820-6436.1$ & 2 & 8.87 & +0.20 & -0.62 & B1 III/IV (1) & \\
\hline 97629 & $-60^{\circ} 2677$ & $111308-6106.8$ & 1 & 9.53 & +0.11 & -0.65 & B3/5 Ib/II (1) & ALS 2250 \\
\hline 98260 & $-60^{\circ} 2783$ & $111719-6130.7$ & 3 & 9.70 & +0.26 & -0.63 & B1/2 Ib/II (1) & ALS 2292 \\
\hline 99024 & $-59^{\circ} 3411$ & $112303-6031.2$ & 1 & 9.66 & +0.03 & -0.77 & B2 Ib/II (1) & ALS 2327 \\
\hline 99205 & $-69^{\circ} 1537$ & $112356-7008.0$ & 2 & 9.59 & +0.01 & -0.73 & B1 III (1) & ALS 2336 \\
\hline 100355 & $-61^{\circ} 2391$ & $113216-6232.8$ & 3 & 9.23 & 0.00 & -0.67 & B2/3 III (1) & \\
\hline 101794 & $-61^{\circ} 2541$ & $114225-6228.6$ & 2 & 8.68 & +0.06 & -0.74 & $\mathrm{~B} 0 / 1 \mathrm{n}(1)$ & $\begin{array}{l}\text { ALS 2460, eclipsing, Be star, } \\
\text { in Stock } 14\end{array}$ \\
\hline 101838 & $-61^{\circ} 2550$ & $114249-6233.9$ & 1 & 8.42 & +0.02 & -0.81 & B0.5/1 III (1) & ALS 2463, eclipsing, in Stock 14 \\
\hline 102505 & $-69^{\circ} 1588$ & $114720-7025.0$ & 2 & 9.02 & +0.08 & - & B1 IV (1) & ALS 2495 \\
\hline 103007 & $-61^{\circ} 2696$ & $115118-6159.8$ & 3 & 9.29 & -0.01 & -0.82 & B2/3 (III) (1) & ALS 2519 \\
\hline 103320 & $-59^{\circ} 3910$ & $115341-6016.0$ & 1 & 8.82 & -0.03 & - & B2 III (1) & \\
\hline 103764 & $-61^{\circ} 2814$ & $115652-6236.6$ & 1 & 9.48 & -0.06 & -0.80 & B2 II/III (1) & \\
\hline 104257 & $-65^{\circ} 1757$ & $120012-6604.7$ & 1 & 8.85 & +0.05 & -0.73 & B1/2 II/III (1) & ALS 2562 \\
\hline 104465 & $-62^{\circ} 2526$ & $120137-6333.8$ & 2 & 9.07 & -0.05 & -0.81 & B2 II/III (1) & \\
\hline 104795 & $-58^{\circ} 4048$ & $120400-5843.4$ & 1 & 9.09 & +0.11 & - & B2 III (1) & \\
\hline 106345 & $-67^{\circ} 1921$ & $121416-6744.4$ & 1 & 9.08 & - & - & B2 III (1) & \\
\hline 108628 & $-61^{\circ} 3220$ & $122906-6228.0$ & 2 & 9.16 & - & - & B2 II (1) & \\
\hline 108769 & $-33^{\circ} 3261$ & $123003-3429.9$ & 1 & 9.03 & - & - & B2 III (3) & \\
\hline 110498 & $-60^{\circ} 4253$ & $124316-6138.9$ & 1 & 9.67 & +0.49 & -0.49 & B0.5 III (1) & ALS 2737, in Cen OB1 \\
\hline 111377 & $-60^{\circ} 4309$ & $124938-6057.9$ & 1 & 9.52 & +0.13 & -0.68 & B2/5 II-III (1) & ALS 2775 \\
\hline 111578 & $-59^{\circ} 4488$ & $125057-6026.8$ & 1 & 9.13 & +0.16 & -0.57 & B2/3 II (1) & ALS 2785 \\
\hline 113013 & $-59^{\circ} 4653$ & $130141-6026.4$ & 2 & 9.40 & +0.12 & - & B2 III (1) & ALS 2871 \\
\hline 114444 & $-74^{\circ} 1029$ & $131304-7518.9$ & 2 & 10.32 & -0.02 & -0.80 & B1 II/III (1) & ALS 2963 \\
\hline 114733 & $-57^{\circ} 5952$ & $131330-5821.7$ & 1 & 9.52 & +0.27 & -0.55 & B2/3 III (1) & ALS 2980 \\
\hline 115533 & $-61^{\circ} 3586$ & $131901-6153.7$ & 1 & 10.04 & +0.08 & -0.76 & $\mathrm{~B} 2 / 5(\mathrm{Ib} / \mathrm{II})(1)$ & ALS 3026, Vis. double, Sep. 6" \\
\hline 116538 & $-51^{\circ} 6048$ & $132512-5150.5$ & 1 & 7.90 & -0.06 & -0.89 & B2 Ib/II (2) & \\
\hline 116827 & $-61^{\circ} 3706$ & $132735-6207.1$ & 2 & 9.51 & +0.11 & -0.57 & $\mathrm{~B} 2 / 5(\mathrm{Vn})(1)$ & Be star \\
\hline 117357 & $-61^{\circ} 3760$ & $133116-6144.0$ & 2 & 9.02 & +0.23 & -0.72 & O9.5/B0 V (1) & ALS 3103, Be star \\
\hline 117687 & $-60^{\circ} 4759$ & $133330-6126.8$ & 1 & 9.33 & +0.12 & -0.71 & $\mathrm{~B} 2 \mathrm{Ib} / \mathrm{II}(1)$ & ALS 3115 \\
\hline 117704 & $-61^{\circ} 3793$ & $133337-6219.1$ & 1 & 8.89 & +0.20 & -0.59 & B1/2 III (1) & ALS 3116 , close to WR 55 \\
\hline 119252 & $-47^{\circ} 6162$ & $134309-4748.3$ & 1 & 10.34 & - & - & B3 III (2) & \\
\hline 119910 & $-60^{\circ} 4978$ & $134754-6053.0$ & 2 & 8.31 & -0.07 & - & B2 III/IV (1) & \\
\hline 122831 & $-67^{\circ} 2491$ & $140701-6834.1$ & 2 & 9.60 & +0.05 & - & B1 III (1) & ALS 3196 \\
\hline
\end{tabular}


Table 1. continued.

\begin{tabular}{|c|c|c|c|c|c|c|c|c|}
\hline HD & $\mathrm{CPD} / \mathrm{BD}$ & ASAS name & $N$ & $\begin{array}{c}V \\
{[\mathrm{mag}]}\end{array}$ & $\begin{array}{l}B-V \\
{[\mathrm{mag}]}\end{array}$ & $\begin{array}{l}U-B \\
{[\mathrm{mag}]}\end{array}$ & MK sp. type & Notes \\
\hline 123077 & $-42^{\circ} 6537$ & $140637-4327.8$ & 4 & 10.30 & -0.23 & - & B2/3 II (2) & \\
\hline 126357 & $-59^{\circ} 5569$ & $142709-5944.2$ & 3 & 9.07 & +0.22 & -0.64 & B1 III (1) & ALS 3238, in NGC 5606 \\
\hline 131805 & $-73^{\circ} 1416$ & $150055-7347.7$ & 1 & 8.47 & - & - & B5 V (1) & long-term variability superimposed \\
\hline 132320 & $-59^{\circ} 5775$ & $150106-5953.7$ & 1 & 9.40 & +0.31 & -0.53 & B1 III (1) & \\
\hline 137405 & $-60^{\circ} 5814$ & $152854-6048.1$ & 1 & 9.36 & +0.13 & -0.64 & B0.5/1 Iab (1) & ALS 3356 \\
\hline 142754 & $-40^{\circ} 7155$ & $155749-4059.8$ & 1 & 8.60 & +0.17 & -0.60 & B2 III (2) & \\
\hline 145537 & $-34^{\circ} 6468$ & $161255-3439.3$ & 2 & 10.41 & +0.10 & -0.78 & B2 Ib/II (3) & \\
\hline 146442 & $-45^{\circ} 7845$ & $161823-4550.4$ & 3 & 9.04 & +0.30 & -0.51 & B2 III (2) & \\
\hline 147421 & $-53^{\circ} 7928$ & $162445-5327.8$ & 1 & 9.02 & +0.09 & -0.81 & B0 II (1) & ALS 3596 \\
\hline 149100 & $-53^{\circ} 8064$ & $163521-5338.9$ & 1 & 7.19 & -0.06 & -0.76 & B2 III (1) & \\
\hline 150927 & $-37^{\circ} 6738$ & $164544-3810.1$ & 2 & 9.44 & +0.32 & -0.52 & $\mathrm{~B} 2 / 3 \mathrm{Ib}(3)$ & \\
\hline 151158 & $-42^{\circ} 7512$ & $164734-4252.7$ & 1 & 8.20 & +0.22 & -0.49 & B2 Ib/II (2) & ALS 3754 \\
\hline 152060 & $-41^{\circ} 7683$ & $165300-4124.5$ & 2 & 9.58 & +0.06 & -0.73 & B1 III (2) & \\
\hline 152162 & $-45^{\circ} 8186$ & $165354-4600.9$ & 2 & 8.59 & +0.18 & - & B2 III (2) & \\
\hline 152372 & $-48^{\circ} 8913$ & $165517-4848.3$ & 3 & 8.85 & +0.20 & -0.60 & B2 III/IV (2) & \\
\hline 153772 & $-50^{\circ} 9813$ & $170400-5105.0$ & 1 & 8.32 & +0.06 & -0.61 & B2 V (2) & in $\mathrm{R}$ assoc. vdBH81a \\
\hline 154500 & $-34^{\circ} 6724$ & $170721-3428.7$ & 2 & 9.94 & +0.22 & -0.56 & B2 Iab/b (3) & \\
\hline 155407 & $-45^{\circ} 8378$ & $171332-4527.2$ & 1 & 9.52 & - & - & B2 II (2) & \\
\hline 156172 & $-41^{\circ} 7933$ & $171800-4203.6$ & 1 & 8.18 & +0.40 & -0.58 & O9.5/B0.5 Ia/Iab (2) & ALS 4037 \\
\hline 156321 & $-32^{\circ} 4462$ & $171820-3219.6$ & 1 & 8.15 & +0.01 & -0.77 & B2 V (3) & \\
\hline 159792 & $-46^{\circ} 8786$ & $173850-4618.9$ & 3 & 9.43 & +0.15 & -0.63 & B2 II (2) & \\
\hline 161633 & $-46^{\circ} 8909$ & $174900-4656.4$ & 4 & 9.94 & +0.12 & -0.95 & B1 Iab/b (2) & \\
\hline 164188 & $-15^{\circ} 4767$ & $180039-1548.1$ & 1 & 8.67 & +0.19 & -0.71 & B1 Ib/II (4) & ALS 4546 \\
\hline 164741 & $-25^{\circ} 6269$ & 180344-2518.7 & 5 & 9.03 & +0.31 & -0.55 & B2 Ib/II (4) & ALS 4590 \\
\hline 165955 & $-34^{\circ} 7625$ & $180958-3452.1$ & 2 & 9.20 & -0.05 & -0.82 & B2 II/III (3) & ALS 4702, high-velocity star \\
\hline 166304 & $-16^{\circ} 4739$ & $181043-1642.8$ & 3 & 9.33 & +0.19 & -0.63 & B2 II (4) & \\
\hline 167003 & $-33^{\circ} 4963$ & $181442-3308.5$ & 4 & 8.45 & -0.13 & -0.94 & B1 Ib/II (3) & ALS 4801, V4386 Sgr, eclipsing \\
\hline 167451 & $-13^{\circ} 4897$ & $181555-1334.5$ & 1 & 8.24 & +0.78 & -0.29 & B1 Ib (4) & ALS 4844, in Ser OB1 \\
\hline 168015 & $-13^{\circ} 4919$ & $181822-1323.2$ & 2 & 9.11 & +0.36 & - & B3/5 II (4) & ALS 9488 \\
\hline 168050 & $-19^{\circ} 6762$ & 181839-1906.2 & 2 & 9.81 & - & - & B3/5 Ib (4) & eclipsing \\
\hline 168675 & $-17^{\circ} 5154$ & $182143-1753.8$ & 2 & 8.94 & +0.28 & - & B2 Ib/II (4) & ALS 4964 \\
\hline 168750 & $-26^{\circ} 6414$ & $182221-2625.0$ & 3 & 8.27 & +0.08 & -0.74 & B1 Ib (3) & ALS 4969 \\
\hline 169173 & $-17^{\circ} 5179$ & $182408-1751.8$ & 1 & 9.93 & - & - & B3 Ib (4) & \\
\hline 169601 & $-17^{\circ} 5192$ & $182607-1747.3$ & 3 & 9.88 & - & - & B0/1 Iab/b (4) & \\
\hline 171141 & $-46^{\circ} 9389$ & $183516-4556.4$ & 1 & 8.37 & -0.22 & -0.97 & B2 II/III (2) & halo star \\
\hline 171305 & $-04^{\circ} 4497$ & $183416-0448.8$ & 1 & 8.70 & +0.45 & - & B2 II (5) & ALS 9749, in the CoRoT field \\
\hline 171344 & $-13^{\circ} 5039$ & $183457-1352.2$ & 1 & 9.52 & +0.29 & - & B2 Ia (4) & ALS 9758 \\
\hline 172140 & $-29^{\circ} 5634$ & 183948-2920.4 & 5 & 9.95 & -0.05 & -0.89 & B1 II (3) & \\
\hline 172427 & $-10^{\circ} 4749$ & $184032-1043.1$ & 1 & 9.46 & +0.48 & -0.44 & B0.5/1 Ib (5) & ALS 9832, in Sct OB2 \\
\hline 173006 & $-05^{\circ} 4737$ & $184326-0546.8$ & 1 & 10.03 & +0.27 & -0.55 & B0.5 IV (5) & ALS 9902, in the CoRoT field \\
\hline 173502 & $-30^{\circ} 5678$ & $184656-2957.6$ & 4 & 9.73 & -0.10 & -0.91 & B1/2 Ib (3) & halo star \\
\hline 178987 & $-47^{\circ} 9208$ & $191259-4709.7$ & 1 & 9.83 & - & - & B2 II (2) & \\
\hline 180032 & $-14^{\circ} 5352$ & $191531-1355.1$ & 2 & 9.49 & -0.07 & - & B2 III/IV (4) & ALS 10219 \\
\hline 186610 & $-03^{\circ} 4698$ & $194527-0309.1$ & 4 & 9.67 & -0.01 & -0.77 & B2 Ib/II (5) & ALS 10480 , in the CoRoT field \\
\hline 187536 & $-28^{\circ} 7032$ & $195125-2813.5$ & 1 & 9.46 & -0.08 & -0.86 & B3 II (3) & \\
\hline
\end{tabular}

low-amplitude mode of 15 (EY) CMa (Shobbrook et al. 2006). In Fig. 18, we plot phase diagrams for the main modes of BW Vul and the three large-amplitude stars mentioned above. It can be seen that HD 173006 and HDE 328862 also exhibit a standstill although it is not so clearly visible as for BW Vul due to the larger scatter of datapoints. The light curve for BW Vul shown in Fig. 18 was obtained in Strömgren $b$ filter, but it is very similar in shape to that in $V$ (see, e.g., Sterken et al. 1993).

As can be seen in Fig. 17, the periods of modes detected in the new $\beta$ Cephei stars match very well the range covered by already known stars. It seems that the largest amplitudes occur in stars with intermediate periods; for the shortest and the longest periods amplitudes are rather small. This result qualitatively agrees with the recent non-linear calculations of Smolec $\&$ Moskalik (2007) for radial modes in $\beta$ Cephei stars.
There were ten known $\beta$ Cephei stars having modes with periods shorter than $0.1 \mathrm{~d}$, of which six are members of the open cluster NGC 6231 (see, e.g., Balona \& Laney 1995). We detect four next ones, HD 115313, 152060, 165955, and 171141. The shortest period, $0.0686 \mathrm{~d}$, was found in HD 115313. It is interesting to note that HD $152060=$ Braes $122=$ NGC 6231-308 is located $28^{\prime}$ off the centre of NGC 6231. It is thus possible that this star is a member of the cluster or of the Sco OB1 association surrounding NGC 6231.

On the other side of the period range we knew two stars with periods slightly longer than 0.3 days, V986 Oph with period equal to $0.303 \mathrm{~d}$ (Cuypers et al. 1989) and Oo 2299 in the open cluster $\chi$ Persei with period of 0.31788 d (Krzesiński \& Pigulski 1997). We found two stars with periods longer than $0.3 \mathrm{~d}$ (see, however, Sect. 3.4): HD 101838 ( $P=0.31973$ d, a member 


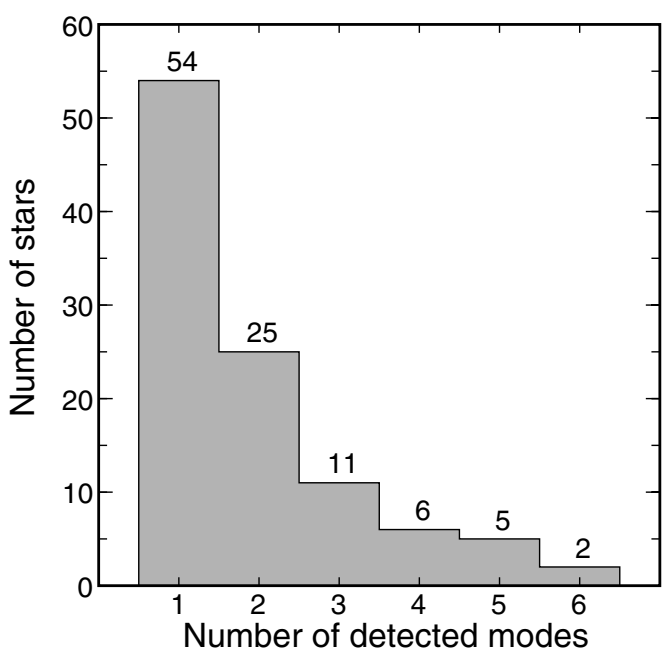

Fig. 15. The histogram of the number of detected modes for the 103 new $\beta$ Cephei-type stars.

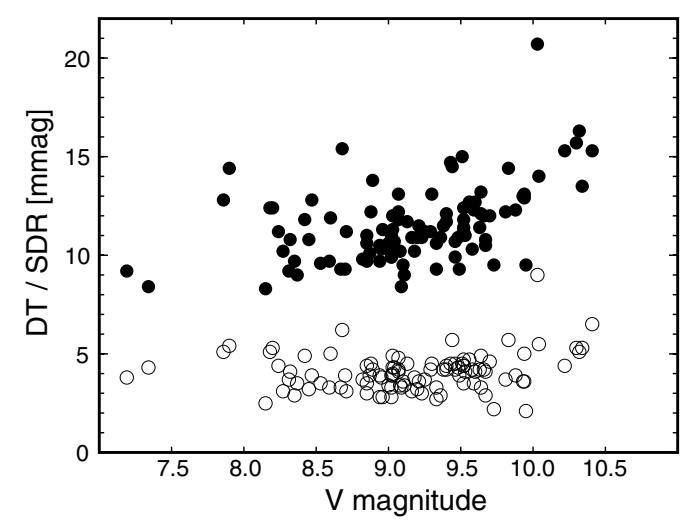

Fig. 16. Detection threshold, DT (open circles), and standard deviation of the residuals, SDR (filled circles), for the ASAS-3 photometry of the 103 new $\beta$ Cephei stars, plotted against their $V$ magnitude. Detection theshold was defined as corresponding to $S / N=4$.

of the open cluster Stock 14 and a component of an eclipsing binary, see Sect. 3.7) and HD $153772=\operatorname{vdBH} 81 \mathrm{a}(P=$ $0.30527 \mathrm{~d}$ ), in the core of the $\mathrm{R}$ association vdBH 81 (van den Bergh \& Herbst 1975; Herbst 1975).

As we already explained in the Introduction, we expected to detect $\beta$ Cephei stars with smaller amplitudes than those found earlier in the ASAS data by Handler (2005) and Pigulski (2005). Figure 17 shows that this is indeed the case.

\subsection{Multiplicity and splittings}

As mentioned in Sect. 3.1 (see also Fig. 15), about half of the new $\beta$ Cephei stars are multiperiodic. In order to show how the modes are spread over the frequencies, in Fig. 19 we plot the differences, $\Delta f$, between frequency of a given mode, $f_{\mathrm{i}}$, and the frequency of the main mode, $f_{1}$, as a function of $f_{1}$. By the main mode we mean the mode with the largest amplitude and period shorter than $0.32 \mathrm{~d}$. We see from Fig. 19 that $\Delta f$ is usually small in comparison with $f_{1}$ and the value of $|\Delta f| / f_{1}$ rarely exceeds 0.2 ; in most cases it is much smaller. There are, however, a few exceptions. First, for HD 126357, the frequencies of two modes, $f_{2}=8.54271 \mathrm{~d}^{-1}$ and $f_{3}=8.07010 \mathrm{~d}^{-1}$, are about twice the frequency of the main mode $\left(f_{1}=4.06927 \mathrm{~d}^{-1}\right)$. This situates these two modes close to the $f_{\mathrm{i}}=2 f_{1}$ line in Fig. 19 . Next, we

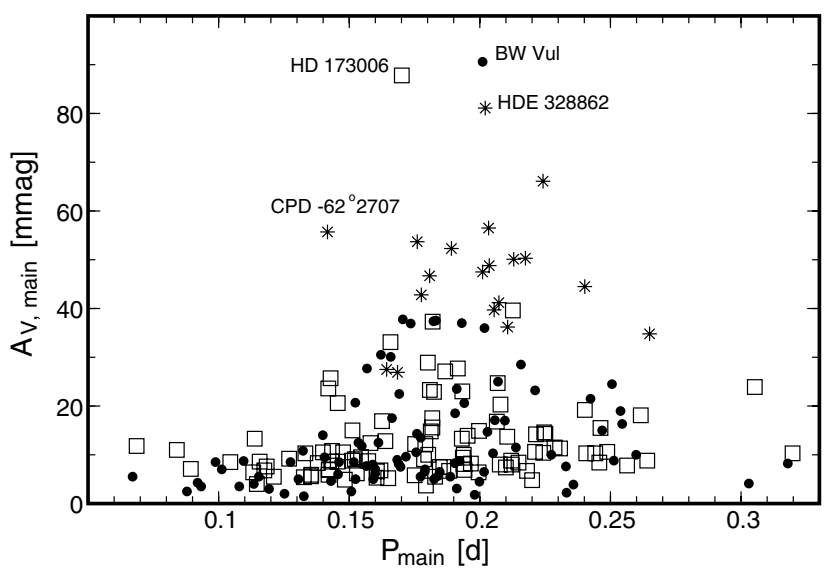

Fig. 17. $V$-filter semi-amplitude of the main mode, $A_{\mathrm{V} \text {,main }}$, plotted as a function of the period of this mode, $P_{\text {main }}$. Black dots denote $\beta$ Cephei stars listed by Stankov \& Handler (2005); $\alpha$ Vir and $\omega^{1}$ Sco are not plotted. Stars discovered by Handler (2005) and Pigulski (2005) are shown with asterisks, and those listed in Table 1, as open squares. The four stars discussed in the text are labelled.

have five stars with modes that are placed below $f_{\mathrm{i}}=f_{1} / 2$ line (asterisks in Fig. 19). We will discuss these stars in Sect. 3.4.

Rotational splittings are observed in some $\beta$ Cephei stars. Good examples are $v$ Eri (Jerzykiewicz et al. 2005), V836 Cen (Aerts et al. 2004), and $\theta$ Oph (Handler et al. 2005). The presence of two modes closely spaced in frequency does not prove that we deal with rotational splitting although this possibility has to be considered in such a case. When three or more equally (or almost equally) spaced modes are observed, the rotational splitting is an obvious conclusion. However, it can happen accidentally that three modes of different $\ell$ form an equidistant triplet, as found for 12 (DD) Lac (Handler et al. 2006).

The rotationally split modes are potentially very useful for mode identification and asteroseismology. We therefore checked the frequencies of the detected modes in the new $\beta$ Cephei stars for possible rotational splittings. The results are summarized in Table 3 and shown in Fig. 20. We list only stars in which inequality of separations is smaller than $20 \%$. The most interesting are the first two stars in Table 20, HD 74339 and HD 86214, those that in our sample have the largest number of detected modes. In both stars equidistant quadruplets are observed. It is quite likely that modes listed in Table 3 are parts of a rotationally split $\ell=1$ or 2 modes. This, however, needs to be verified by mode identification, as was done for $12 \mathrm{Lac}$.

\subsection{Stars with long periods}

The longest periods observed in Galactic $\beta$ Cephei stars rarely exceed $0.3 \mathrm{~d}$ (see Fig. 17) and until recent years there was no clear evidence for the presence of low-frequency $g$ modes in these stars. From the theoretical point of view, however, some overlap of the instability strips of $\beta$ Cephei and slowly pulsating B (SPB) stars (e.g., Pamyatnykh 1999) indicated that some hybrid objects might be found. The recent campaigns on two well-known $\beta$ Cephei stars, $v$ Eri (Jerzykiewicz et al. 2005) and 12 (DD) Lac (Handler et al. 2006), led to the discovery of low-frequency mode(s) in these stars.

On the other hand, Kołaczkowski et al. $(2004,2006)$ announced the discovery of a large number of short-period variable stars in the Magellanic Clouds showing long-period mode(s) (periods in the range of $0.4-1 \mathrm{~d}$ ) in addition to mode(s) 

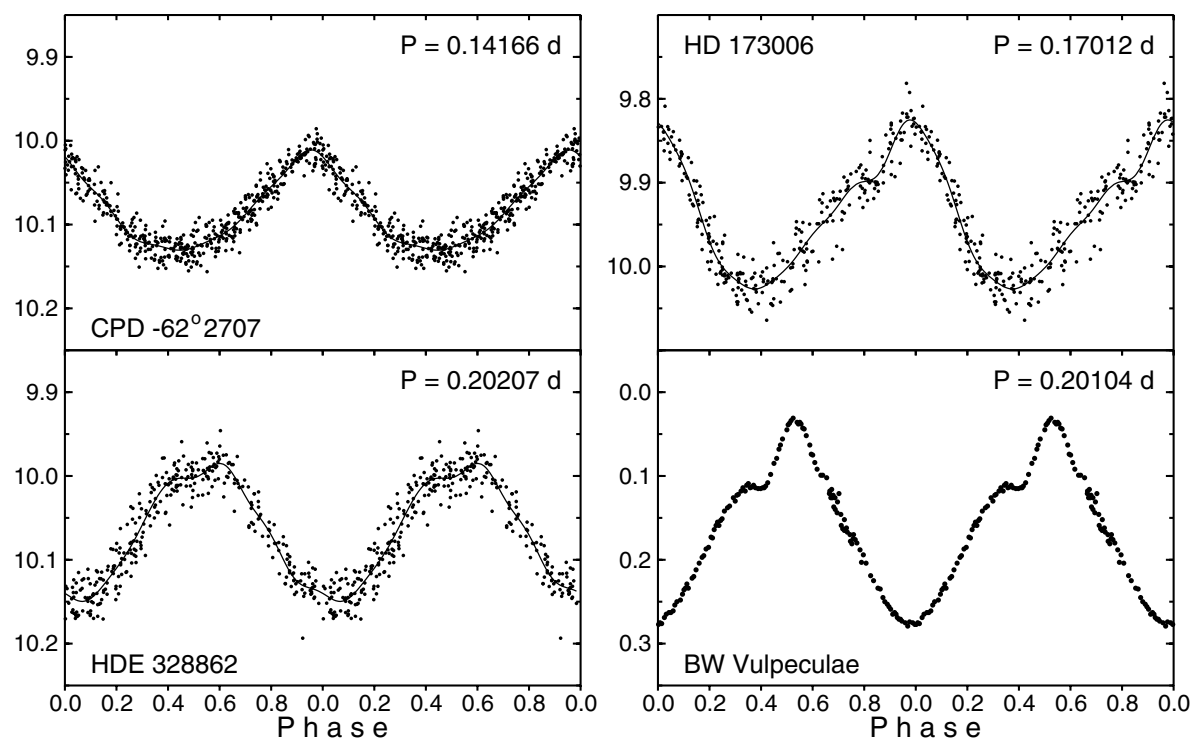

Fig. 18. Light curves of three large-amplitude $\beta$ Cephei stars discovered in the ASAS-3 data and BW Vulpeculae. Continuous lines are fits including the main frequency and five lowest harmonics. For the ASAS-3 stars, the ordinate is the $V$ magnitude, for BW Vul, differential Strömgren $b$ magnitude. Observations of BW Vul were taken from the 1982 campaign data (Sterken et al. 1986).

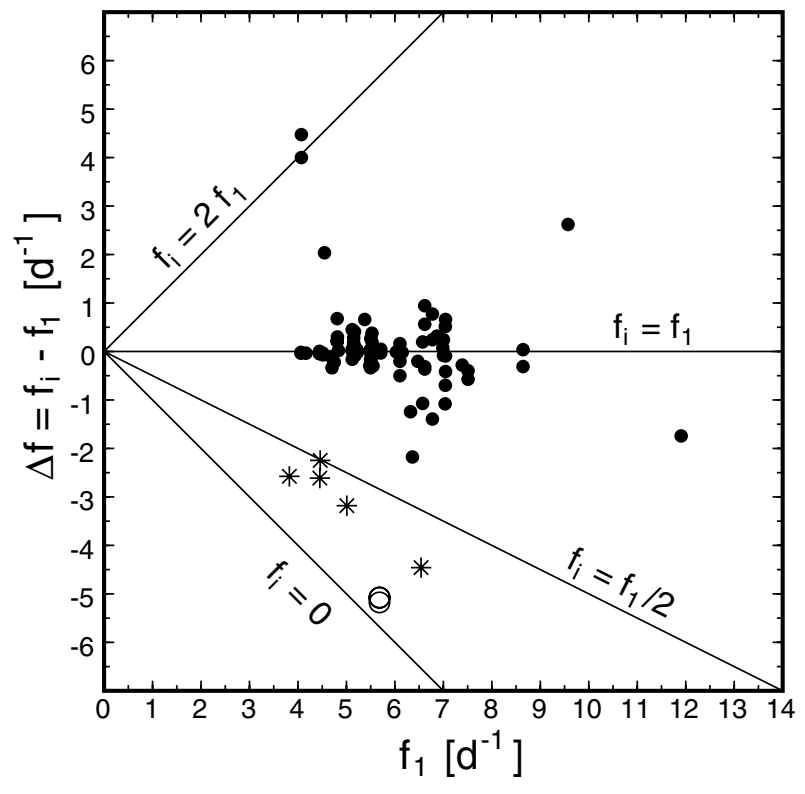

Fig. 19. The difference, $\Delta f=f_{\mathrm{i}}-f_{1}$, where $f_{\mathrm{i}}$ is the frequency of a given mode and $f_{1}$, the frequency of the main mode, for 49 multiperiodic $\beta$ Cephei stars discovered in this paper. Low-frequency modes (see Sect. 3.4) are shown as asterisks. In addition, long-period modes for HD 133823 (Paper I) are shown with open circles.

typical for $\beta$ Cephei stars. The origin of these long-period modes still needs to be explained. We will come back to the discussion of this problem in Sect. 4.

In the ASAS-3 data we have found five stars having periods considerably longer than $0.3 \mathrm{~d}$ (Fig. 19), namely, in the range between 0.45 and $0.80 \mathrm{~d}$. These stars are listed in Table 4 . The most interesting of the five stars listed in Table 4 is HD $101794=$ V916 Cen = Stock14-13. The star is a member of the loose open cluster Stock 14 = Lodén 462/465 (Lodén 1973), although Humphreys (1978) and Kaltcheva et al. (1994) include it to the association Cru OB1. The star was classified as B1 IVne by Garrison et al. (1977). Its Be status was confirmed by measurements of the $\beta$ index (Moffat \& Vogt 1975; Klare \& Neckel 1977; Johansson 1981; Kaltcheva 2003) and H $\alpha$ photometry (McSwain \& Gies 2005).
Table 3. Possible rotational splittings for seven stars from our sample. Designation of frequencies is the same as in Table 2.

\begin{tabular}{rlll}
\hline \hline Star & $\Delta f$ & Splitting $\left[\mathrm{d}^{-1}\right]$ & Note \\
\hline HD 74339 & $f_{3}-f_{5}$ & $0.01690(4)$ & quadruplet \\
& $f_{4}-f_{3}$ & $0.01673(4)$ & \\
& $f_{6}-f_{4}$ & $0.01669(4)$ & \\
HD 86214 & $f_{2}-f_{5}$ & $0.00468(5)$ & quadruplet \\
& $f_{3}-f_{2}$ & $0.00505(4)$ & \\
& $f_{4}-f_{3}$ & $0.00495(4)$ & \\
HD 94065 & $f_{5}-f_{3}$ & $0.00155(4)$ & triplet \\
& $f_{2}-f_{5}$ & $0.00139(4)$ & \\
HD 98260 & $f_{3}-f_{2}$ & $0.03716(4)$ & triplet \\
& $f_{1}-f_{3}$ & $0.03386(4)$ & \\
HD 168750 & $f_{3}-f_{2}$ & $0.01833(2)$ & triplet \\
& $f_{1}-f_{3}$ & $0.01838(4)$ & \\
HD 173502 & $f_{3}-f_{4}$ & $0.17543(9)$ & triplet \\
& $f_{1}-f_{3}$ & $0.16308(3)$ & \\
HD 186610 & $f_{1}-f_{3}$ & $0.03871(4)$ & triplet \\
& $f_{4}-f_{1}$ & $0.03863(5)$ & \\
\hline
\end{tabular}

Table 4. Stars with periods longer than $0.4 \mathrm{~d}$.

\begin{tabular}{cccl}
\hline \hline Star & $\begin{array}{c}\text { Short } \\
\text { period [d] }\end{array}$ & $\begin{array}{c}\text { Long } \\
\text { period [d] }\end{array}$ & Note \\
\hline HD 99205 & 0.26161 & 0.80203 & \\
HD 101794 & 0.22447 & 0.54362 & V916 Cen, Be \\
HD 116827 & 0.22429 & 0.45217 & Be \\
HD 117357 & 0.15291 & 0.48052 & Be \\
HD 122831 & 0.19968 & 0.54730 & \\
\hline
\end{tabular}

HD 101794 was discovered as variable by Hipparcos and was subsequently named V916Cen. The star was assigned the variability type of $\gamma$ Cas, which labels B-type stars showing erratic long-term changes with a range up to $0.5 \mathrm{mag}$. Suprisingly, HD 101794 is also an eclipsing star (see Sect. 3.7). There are at least two other periodic terms superimposed on the variability due to eclipses (Table 4). One of them has a period in the $\beta$ Cephei domain $(P=0.22447 \mathrm{~d})$ and this is the reason for including the star in the list of $\beta$ Cephei stars. Another periodicity, with a period of about $0.54362 \mathrm{~d}$, can be attributed to a $\lambda$ Eridani-type variability. 

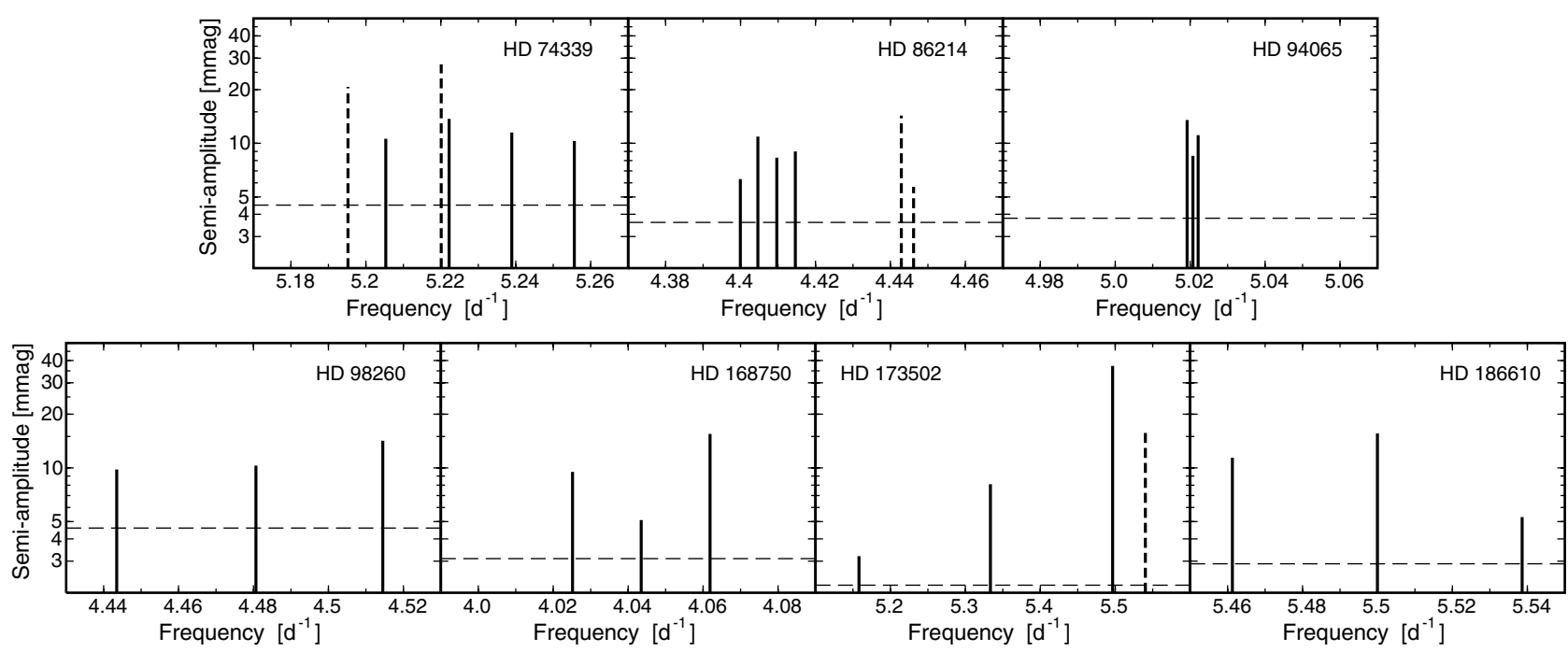

Fig. 20. Schematic frequency spectra in the vicinity of the possible rotationally split modes of seven new $\beta$ Cephei stars. For all stars but HD 173502 the frequency range shown is equal to $0.1 \mathrm{~d}^{-1}$. Modes which are not components of the suggested multiplets are plotted with dashed lines. See also Table 3.

Another star from Table 4, HD 116827, is also known to be a Be star (MacConnell 1981). Houk \& Cowley (1975) classify the star as $\mathrm{B} 2 / 5(\mathrm{Vn})$ and note that the $\mathrm{H} \beta$ line may be partially filled in, a clear indication of a weak emission.

The third new $\beta$ Cephei star with both short and long-period variability, is HD 117357. It shows even stronger emission than HD 101794 in hydrogen Balmer lines (Schmidt-Kaler 1964; Garrison et al. 1977; Wiegert \& Garrison 1998; Yudin 2001). The emission in $\mathrm{H} \beta$ is confirmed by two measurements of the $\beta$ index: 2.522 (Deutschmann et al. 1976) and 2.502 (Klare \& Neckel 1977).

There is no recorded evidence for emission in hydrogen lines for the two remaining stars in Table 4, HD 99205 and HD 122831. Since three of five stars in Table 4 show Balmerline emission and only two of the remaining $98 \beta$ Cephei stars are known as Be stars, it is hard to believe that the occurence of long periods and Balmer-line emission remains unrelated. We will come back to the discussion of the origin of long periods in these stars in Sect. 4.

\subsection{Period and amplitude changes}

Period and amplitude changes are observed in some $\beta$ Cephei stars (Jerzykiewicz \& Pigulski 1998, Paper I); they usually take place on a time scale of years or decades. Because ASAS-3 data cover five years, we may expect detecting period changes only if they are very fast. Indeed, only one star, HD 168050, shows pronounced period changes. The photometry of HD 168050 reveals not only two modes attributable to the $\beta$ Cephei-type variability (Table 2), but also eclipses (see Sect. 3.7), indicating that the pulsating star is a component of an eclipsing binary. For one of the detected pulsation modes, the period is stable, while for the other the period increases rapidly. We show the $\mathrm{O}-\mathrm{C}$ diagram for both modes in Fig. 21. Assuming constant rate of period change for $P_{2}$, we get $\mathrm{d} P_{2} / \mathrm{d} t=104 \pm 8 \mathrm{~s} \mathrm{century}^{-1}$. This is the fastest change of period ever detected in a $\beta$ Cephei-type star. Times of maximum light for both modes of HD 168050 are given in Table 5.

As far as the long-term amplitude changes are concerned, we found no clear evidence for them among the newly discovered $\beta$ Cephei stars.

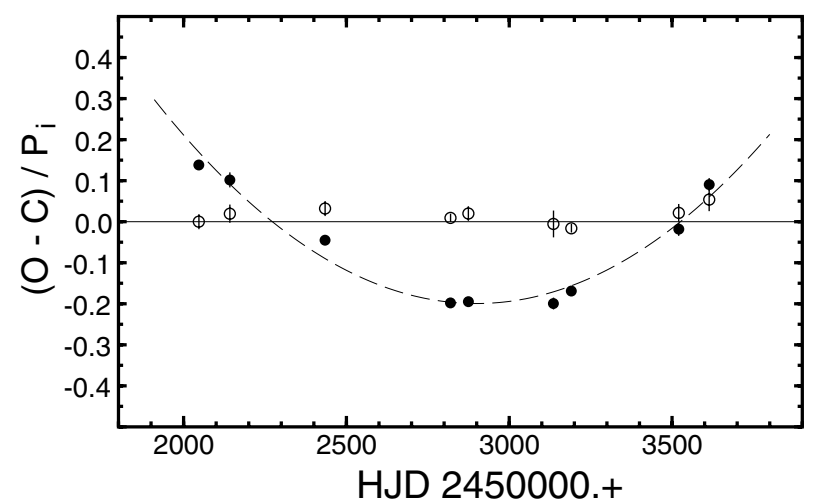

Fig. 21. The O-C diagram for both modes detected in HD 168050. The dashed line represents constant rate of period change equal to 104 s century $^{-1}$.

Table 5. Time of maximum light for both modes detected in HD 168050.

\begin{tabular}{|c|c|}
\hline \multicolumn{2}{|c|}{$\left.{ }_{\text {max, } 1}^{1} I_{\max } 2450000.0+\right]$} \\
\hline $2047.1099(20)$ & 2047.043 \\
\hline 2 & 142.07 \\
\hline 243 & 2434.590 \\
\hline 2819.86 & 2819. \\
\hline 2874.652 & 2874.50 \\
\hline 3135.9806 & 3135.96 \\
\hline 3190.9553(1 & 19) \\
\hline 3520.4 & 3520 \\
\hline 3613 & $.9773(51)$ \\
\hline
\end{tabular}

\subsection{O-type stars}

Although B0 is the earliest spectral type of known $\beta$ Cephei stars, from the theoretical point of view the instability strip of $\beta$ Cephei stars extends to O-type stars (see, e.g., Pamyatnykh 1999). The searches for $\beta$ Cephei-type pulsations in O-type stars, both in the Galactic field (Balona 1992) and Cygnus OB2 association (Pigulski \& Kołaczkowski 1998), were not conclusive, however. On the other hand, line-profile variations were 
observed in some fast-rotating late O-type stars such as $\zeta$ Oph (Vogt \& Penrod 1983; Kambe et al. 1990). They were usually attributed to pulsations in modes with relatively high $\ell$, undetectable in photometry. A recent MOST satellite photometry of $\zeta$ Oph (Walker et al. 2005a) clearly revealed $\beta$ Cephei-type pulsations. It is therefore reasonable to regard $\zeta$ Oph and several other late O-type stars showing non-radial pulsations in line profiles (Fullerton et al. 1996; Balona \& Dziembowski 1999) as $\beta$ Cephei stars.

Among the new $\beta$ Cephei stars, there are at least two O-type stars. The first one is HD 91651 = HIP 51681, a member of Car OB1 association. Its published MK spectral types are the following: O9 Vp? (Morgan et al. 1955), O9 Vn: (Feast et al. 1957), O9 V:n (Walborn 1973), O8/9 V (Houk \& Cowley 1975), O9 Vnp (Garrison et al. 1977), and O9.5 III (Mathys 1988). The star is fast-rotating, the projected radial velocity amounting 280-300 km s${ }^{-1}$ (Penny 1996; Howarth et al. 1997; Penny et al. 2004). The star is known to have strong nitrogen lines for its spectral type (Garrison et al. 1977; Schild 1985; Howarth \& Prinja 1989). The variability of radial velocity of HD 91651 was indicated by Levato et al. (1988) and Lehner et al. (2003). Levato et al. (1988) suspected even that the star is a double-lined spectroscopic binary with an orbital period of $9.275 \mathrm{~d}$. It is interesting to note that from the study of line profiles in this star, Penny (1996) already suggested the possibility of non-radial pulsations.

The other O-type star is HD 156172 with two published spectral types: O9.5/B0.5 Ia/Iab (Houk 1978) and O9 Vnne (Garrison et al. 1977). The star is located in the region of the HI bubble surrounding O5 Vn((f)) star HD 155913, and could belong to an unknown association (Cappa \& Benaglia 1998).

Two other stars are classified as $09 / \mathrm{B} 0 \mathrm{~V}$ and $09.5 / \mathrm{B} 0 \mathrm{~V}$ by Houk \& Cowley (1975), namely, HD 95568 and HD 117357. For HD 95568 this is the only MK spectral type available, for HD 117357 there are several other classifications indicating that it is rather a fast-rotating B0 star.

Although we cannot exclude that variability we detected in O-type stars is due to the pulsations of their, presently unknown, early B-type companions, this seems to be rather unlikely.

\subsection{Components of detached eclipsing binaries}

If a $\beta$ Cephei star is a component of an eclipsing system, one has an opportunity of deriving its accurate mass and radius. Of the 112 previously known $\beta$ Cephei stars, there were four components of eclipsing systems (see Pigulski 2006): 16 (EN) Lac (Jerzykiewicz et al. 1978; Pigulski \& Jerzykiewicz 1988), V381 Car in NGC 3293 (Engelbrecht \& Balona 1986; Jerzykiewicz \& Sterken 1992; Freyhammer et al. 2005), $\eta$ Ori (Kuntz \& Stebbins 1916; Waelkens \& Lampens 1988; De Mey et al. 1996), and $\lambda$ Sco (Shobbrook \& Lomb 1972; Uytterhoeven et al. 2004; Bruntt \& Buzasi 2006).

We add four stars to this list. Surprisingly, two are members of a loose open cluster Stock 14 (Moffat \& Vogt 1975; FitzGerald \& Miller 1983; Peterson \& FitzGerald 1988): HD 101794 and HD 101838. The other two are HD 167003 and HD 168050. It is interesting to note that at least in two stars, HD 101794 and HD 168050 the secondary eclipses are deep enough to expect that spectroscopically they will be double-lined systems. If this were the case, the follow-up studies should allow deriving the masses and radii of the components.

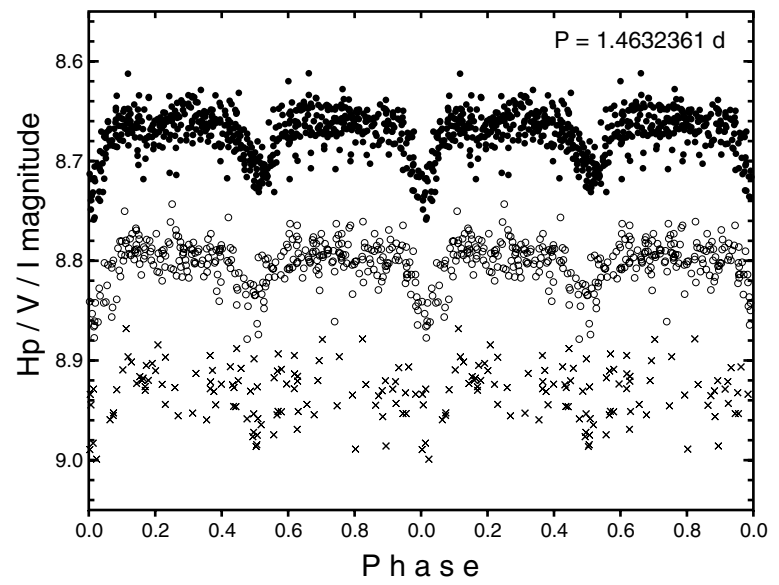

Fig. 22. The eclipsing light curve of V916 Cen folded with the orbital period of $1.4632361 \mathrm{~d}$ for ASAS-2 I-filter data (dots), ASAS-3 $V$-filter data (open circles) and Hipparcos $H_{\mathrm{p}}$ data (crosses). The long-term changes and the contribution from pulsation were removed.

\subsubsection{HD $101794=$ ALS $2460=$ HIP $57106=$ V916 Cen $=$ Stock 14-13}

As mentioned in Sect. 3.4, photometric variability of HD 101794, a Be star and a member of Stock 14, was discovered by the Hipparcos satellite. Its eclipsing nature was revealed by Pojmański (2000) with the I-filter ASAS-2 data. The orbital period amounted to $1.4632 \mathrm{~d}$. As already explained in Sect. 3.4, the star shows also low-amplitude periodic variations. The eclipsing light curve of HD 101794 is shown in Fig. 22. We see that after removing the contribution from the long-term and sinusoidal variations, the eclipses are clearly seen both in the 1997-1999 ASAS-2 data and the ASAS-3 data. Although barely, they can be even seen in the Hipparcos data. The ephemerides for eclipses of V916 Cen and the other three stars are given in Table 6.

\subsubsection{HD $101838=$ ALS $2463=$ Stock $14-14$}

HD 101838 is also a member of the same cluster, Stock 14. Like in V916 Cen, presumably the primary component is a $\beta$ Cepheitype star. The pulsation period revealed from the ASAS-3 photometry is quite long, amounting to $0.31973 \mathrm{~d}$. The star is classified as B1 III (Feast et al. 1961; Walker 1963), B0.5/1 III (Houk \& Cowley 1975), B1 II-III (Garrison et al. 1977), or B0 III (FitzGerald \& Miller 1983).

The eclipsing light-curve of HD 101838 is shown in Fig. 23. When folded with the period of $5.41166 \mathrm{~d}$, it shows no sign of a secondary minimum. However, we cannot exclude the possibility that the true orbital period is twice as long, i.e., it is equal to about $10.82 \mathrm{~d}$. If this were the case, the system would consist of two similar components.

\subsubsection{HD $167003=$ ALS $4801=$ HIP $89404=$ V4386 Sgr}

The variability of HD 167003 was detected by the Hipparcos satellite. Since five observational points obtained within half a day deviated by about 0.2 mag from the remaining observations, it was concluded that the star is possibly an eclipsing binary. The star was classified as an unsolved variable and named V4386 Sgr. There were about a dozen or so deviating points in the ASAS-3 data, clearly indicating the presence of a narrow eclipse. Fortunately, the ASAS-3 data are better distributed in time than those from Hipparcos, allowing to unambiguously 
Table 6. Data for the four new $\beta$ Cephei stars in eclipsing systems.

\begin{tabular}{cccl}
\hline \hline HD & $P_{\text {orb }}[\mathrm{d}]$ & HJD of $T_{\min , 0}$ & Remarks \\
\hline 101794 & $1.4632361(21)$ & $2450561.9213(15)$ & V916 Cen, Be star, member of Stock 14 \\
101838 & $5.41166(7)$ & $2452014.633(13)$ & member of Stock 14 \\
167003 & $10.79824(11)$ & $2452692.824(11)$ & V4386 Sgr \\
168050 & $5.02335(6)$ & $2452096.224(10)$ & \\
\hline
\end{tabular}

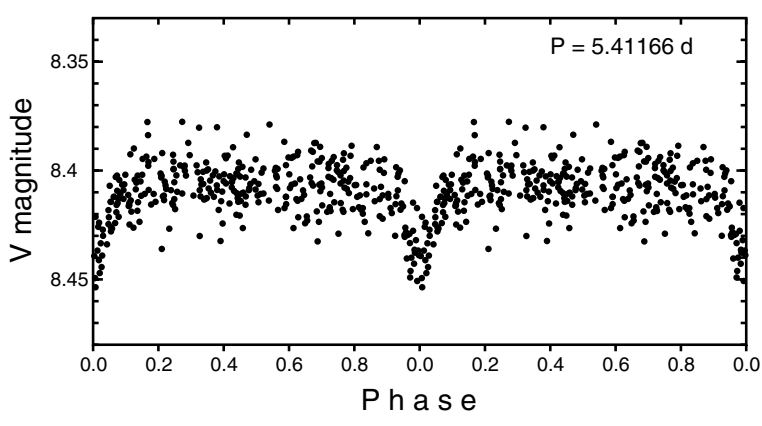

Fig. 23. The ASAS-3 $V$-filter light-curve of HD 101838, freed from the contribution of pulsation and folded with the orbital period of $5.41166 \mathrm{~d}$.
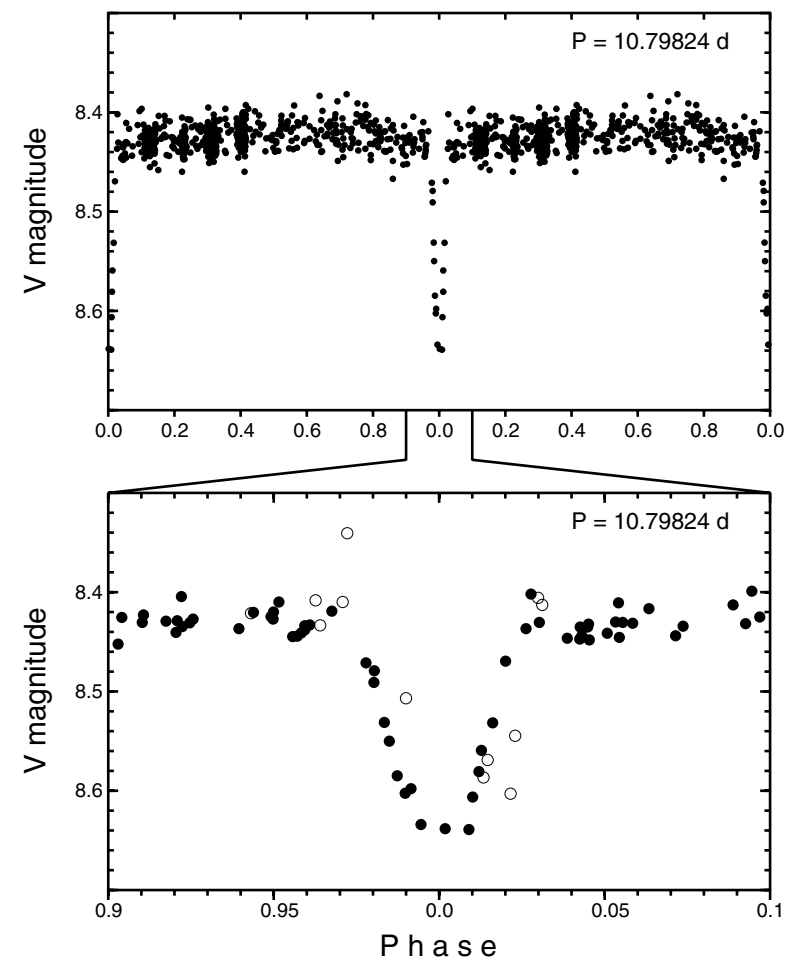

Fig. 24. The ASAS-3 $V$-filter light curve of HD 167003 (V4386 Sgr) freed from the contribution of pulsations and folded with the orbital period of $10.79824 \mathrm{~d}$. The upper figure shows the whole light curve, the lower, only phases close to the primary minimum. In addition, Hipparcos data (not corrected for the contribution from pulsations) are plotted in the lower figure as open circles.

derive the orbital period. The period is equal to $10.79824 \mathrm{~d}$. The light curve (Fig. 24) shows also a small reflection effect. The analysis of the out-of-eclipse data reavealed the presence of at least four pulsational components with frequencies between 5.38 and $7.55 \mathrm{~d}^{-1}$ (Table 2), indicating clearly that the primary component is a $\beta$ Cephei-type pulsator. This is in agreement with the published MK spectral types of this star: B0.5 III (Hill et al. 1974), B1 II (Garrison et al. 1977), and B1 Ib/II (Houk 1978).

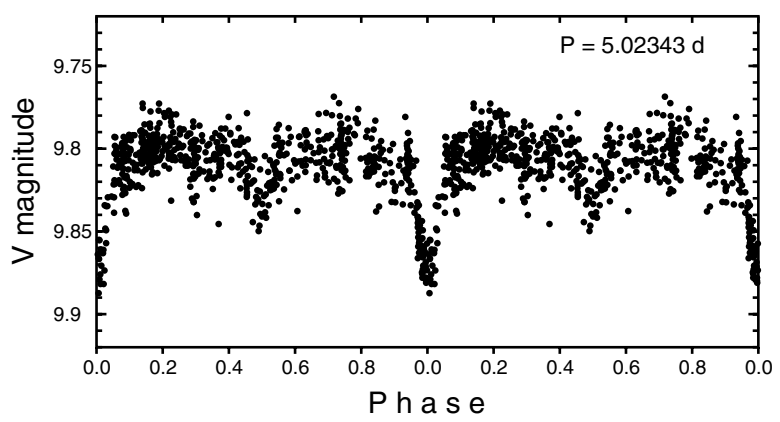

Fig. 25. The ASAS-3 $V$-filter light curve of HD 168050 freed from the contribution of two pulsating modes and folded with the orbital period of $5.02343 \mathrm{~d}$.

As can be seen in Fig. 24, the eclipse covers only about 0.04 in phase, corresponding to about 10 hours, and is probably total. In the lower panel of Fig. 24, Hipparcos data obtained close to the primary minimum are also plotted. The five deviating points mentioned earlier fit the eclipse quite well. However, due to the small number of data points, the contribution from pulsations could not be removed from the Hipparcos data. This may account for the deviations with respect to the ASAS-3 light curve (dots).

\subsubsection{HD 168050}

The fourth $\beta$ Cephei star we discovered in an eclipsing system is HD 168050. From the analysis of the ASAS-3 data we found two periodic terms with frequencies 5.549 and $5.251 \mathrm{~d}^{-1}$, certainly coming from pulsations. It is interesting to note that the first mode shows large period increase (see Sect. 3.5), a phenomenon which is rather rare among $\beta$ Cephei stars. The eclipsing lightcurve of this star is shown in Fig. 25. The orbital period is equal to $5.02335 \mathrm{~d}$ and the eclipses are rather shallow. The depth of the secondary minimum is about $1 / 3$ of that of the primary one which indicates that the secondary component is relatively massive. This also means that the contribution of the secondary to the spectrum of the system is significant and may explain why the spectral type given by Houk \& Smith-Moore (1988), B3/5 Ib, is slightly too late for a $\beta$ Cephei-type star.

\subsection{Distribution in Galactic coordinates}

Since $\beta$ Cephei stars are very young, we expect to find them rather close to the Galactic plane. As discussed by Pigulski (2005), the known stars of this type are indeed located at low Galactic latitudes, except for a few nearby stars and an interesting high-latitude star, HN Aqr (Waelkens \& Rufener 1988; Lynn et al. 2002). A strip of stars belonging to the Gould Belt could be also identified. However, the observed distribution was biased due to observational selection effects. The homogeinity of the ASAS-3 data and the Michigan catalogues allows studying this distribution quantitatively. As can be seen in Fig. 26, the 


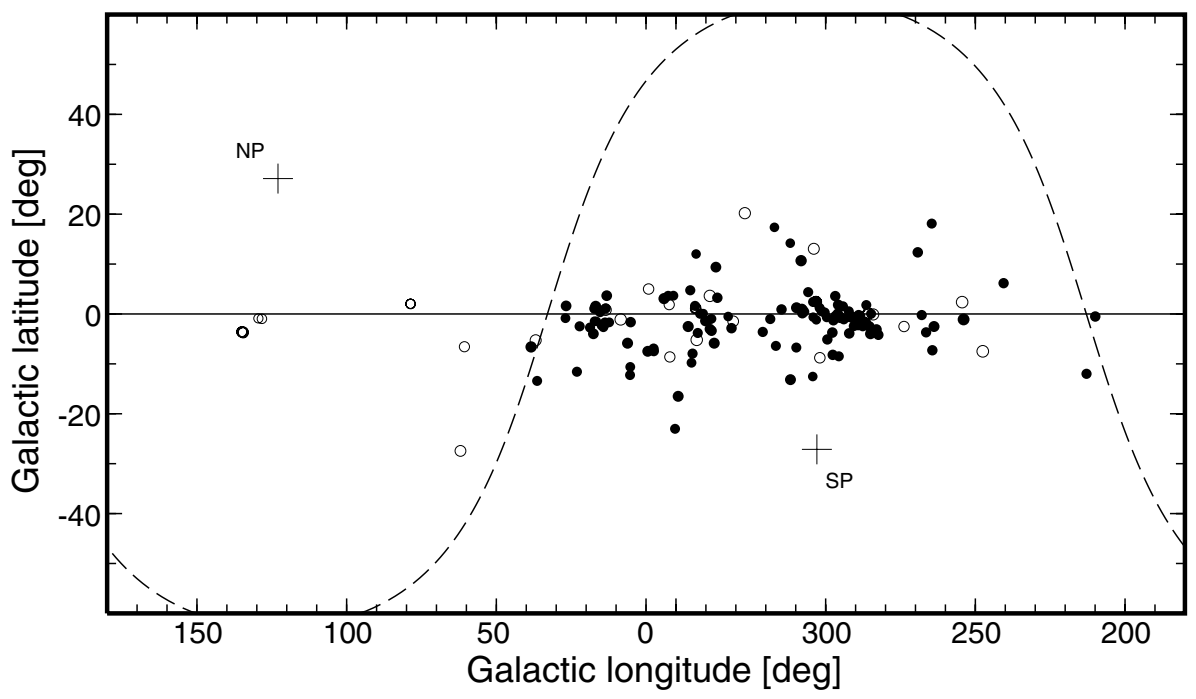

Fig. 26. Distribution of $\beta$ Cephei stars in Galactic coordinates. Only stars with $V$ magnitudes between 7 and $11 \mathrm{mag}$ are shown. Open circles denote stars from Stankov \& Handler (2005), filled circles, stars found in the ASAS-3 data. "NP" and "SP" indicate the northern and southern celestial pole, respectively. Dashed line is the celestial equator. new members of the $\beta$ Cephei family are distributed very unevenly in Galactic coordinates: they tend to form at least three large groups. The largest group, with a center approximately at $(l, b)=\left(300^{\circ}, 0^{\circ}\right)$ agrees with the direction in which we look along the Sagittarius-Carina spiral arm. We postpone a detailed discussion of this distribution until a future paper, in which the data for the remaining $\beta$ Cephei stars will be given. We just indicate here that there are several stars with quite large Galactic latitudes which, taking into account their absolute magnitudes and low reddenings, are situated at large distances both from the Sun and from the Galactic plane. Their distances might be comparable to the distance of the Galactic center, while the distance from the Galactic plane might be as large as $1 \mathrm{kpc}$ or even larger.

\subsection{Stars in open clusters and associations}

We have already indicated in Paper I that owing to the poor spatial resolution, the ASAS-3 data are not well suited for the detection of $\beta$ Cephei stars in open clusters. However, if a cluster is sparse or the variable is far from the central area of the cluster, the ASAS-3 photometry can allow detection. In the sample of the 103 new $\beta$ Cephei stars we can indicate six stars which are members or likely members of young open clusters, and other six which are members of OB associations. This is indicated in the last column of Table 1 . The membership of new $\beta$ Cephei stars in clusters and their distribution in the Galaxy will be discussed in the next paper of the series.

\subsection{Hipparcos stars}

The original work on Hipparcos data resulted in the discovery of six $\beta$ Cephei stars (Waelkens et al. 1998; Aerts 2000). Later on, some follow-up work was done (Koen 2001; Koen \& Eyer 2002) yielding about 60 candidates for short-period variables among $\mathrm{O}$ and early B-type stars of which at least some are expected to be $\beta$ Cephei stars. Due to the unfavourable distribution of the Hipparcos data in time, this discovery needs, however, to be confirmed by independent observations. With the ASAS-3 data, we could verify the variability of 22 of these candidates; they are listed in Table 7. The remaining stars are either saturated in the ASAS-3 data or are located north of declination $+28^{\circ}$, i.e., outside the area in the sky covered by the ASAS-3 observations.

Of these 22 stars, only seven can be classified as $\beta$ Cephei stars. One, HD 191531, was already discovered by
Handler (2005), see also Paper I. The remaining six are new $\beta$ Cephei stars, but only four are listed in Table 1, while two, HDE 306387 = V909 Cen and HDE 339483, are not included because they do not appear in the Michigan catalogues. These two stars will be discussed in the next paper of the series.

For the remaining 15 stars from Table 7, the short-period variability was not confirmed by the ASAS-3 data, although for all but HD 42597 the detection threshold was smaller than the amplitude reported from the analysis of the Hipparcos data. It can be seen from Table 7 that frequencies for most of them range between 10 and $12 \mathrm{~d}^{-1}$. As already explained by Jerzykiewicz \& Pamyatnykh (2000) and Koen \& Eyer (2002), these spurious frequencies are due to aliasing originating from the convolution of the low-frequency changes and the spectral window dominated by the rotation frequency of the satellite $\left(11.25 \mathrm{~d}^{-1}\right)$.

For five of the seven $\beta$ Cephei stars in Table 7, the agreement between frequencies derived from the Hipparcos and ASAS-3 data is very good. Only for HD 68962 and 86065 the differences are significant, amounting to about $\Delta f=f_{\mathrm{ASAS}}-f_{\mathrm{Hipp}}=$ -0.0749 and $+0.0203 \mathrm{~d}^{-1}$, respectively. This can be explained in terms of aliasing in the periodograms of Hipparcos data.

\section{Discussion and conclusions}

According to theory (see, e.g., Pamyatnykh 1999), for the zeroage main-sequence models of massive stars, acoustic $(p)$ and gravity $(g)$ modes separate very well in frequency. This separation is not so good for evolved stars because in the course of the main-sequence evolution frequencies of $g$ modes increase and eventually replace those of $p$ modes through avoided crossing (Dziembowski \& Pamyatnykh 1993). Such modes have a "mixed" character: of a $p$ mode in the envelope and of a $g$ mode in the stellar interior. When only unstable modes are considered, however, the separation in frequency of $p$ and $g$ modes is, for a given model, preserved. Pulsations in $p$ modes and mixed modes are attributed to $\beta$ Cephei-type variability, whereas those in $g$ modes, to the SPB type. As $\beta$ Cephei stars populate mainly the later stages of main-sequence evolution, mixed modes are expected to be excited in these stars.

Theoretical calculations show also (Pamyatnykh 1999) that $\beta$ Cephei and SPB instability strips partially overlap, which means that hybrid stars, showing pulsations of both types, might exist. This possibility was already indicated by Dziembowski et al. (1993) for a region around spectral type B2-B3 and masses 
Table 7. The results of the verification of variability of $22 \mathrm{O}$ and early B-type candidate short-period variables observed by Hipparcos and found by Koen (2001) and Koen \& Eyer (2002). DT is the detection threshold in the ASAS-3 photometry.

\begin{tabular}{|c|c|c|c|c|c|c|c|c|c|c|}
\hline HIP & $\mathrm{HD}(\mathrm{E})$ & $\begin{array}{l}\text { ASAS } \\
\text { name }\end{array}$ & $\begin{array}{c}V \\
{[\mathrm{mag}]}\end{array}$ & $\begin{array}{l}f_{\text {Hipp }} \\
{\left[\mathrm{d}^{-1}\right]}\end{array}$ & $\begin{array}{c}A_{\text {Hipp }} \\
{[\mathrm{mmag}]}\end{array}$ & $\begin{array}{l}f_{\text {ASAS }} \\
{\left[\mathrm{d}^{-1}\right]}\end{array}$ & $\begin{array}{c}A_{\mathrm{ASAS}} \\
{[\mathrm{mmag}]}\end{array}$ & $S / N$ & $\begin{array}{r}\text { DT } \\
{[\mathrm{mmag}]}\end{array}$ & Remarks \\
\hline 29429 & 42597 & $061159+0723.5$ & 7.04 & 10.67562 & 6.7 & - & - & - & 8.0 & \\
\hline 30393 & 44597 & $062329+2023.5$ & 9.04 & 3.25095 & 9.9 & - & - & - & 7.3 & \\
\hline 35355 & 56847 & $071810-1537.7$ & 8.92 & 10.68006 & 26.4 & - & - & - & 10.3 & MU CMa \\
\hline 36727 & 60308 & $073313-1527.2$ & 8.20 & 11.20005 & 16.9 & - & - & - & 9.1 & \\
\hline 38896 & 65658 & $075738-4635.6$ & 7.23 & 11.76946 & 7.2 & - & - & - & 4.3 & \\
\hline 40268 & 68962 & $081323-3618.7$ & 7.34 & 4.83740 & 7.6 & 4.76251 & 7.4 & 6.9 & 4.3 & new $\beta$ Cephei star \\
\hline 46876 & 82830 & 093309-4645.9 & 9.23 & 7.35793 & 16.9 & - & - & - & 9.2 & \\
\hline 48652 & 86085 & $095519-3845.8$ & 8.93 & 7.85499 & 11.6 & 7.87529 & 9.2 & 9.4 & 3.9 & new $\beta$ Cephei star \\
\hline 50598 & 89767 & $102012-5236.1$ & 7.21 & 11.08927 & 12.6 & - & - & - & 8.4 & V342 Vel \\
\hline 55499 & 306387 & $112159-6101.8$ & 9.58 & 4.80836 & 31.6 & $\begin{array}{l}4.80836 \\
4.99402\end{array}$ & $\begin{array}{l}34.5 \\
14.6\end{array}$ & $\begin{array}{l}26.5 \\
11.2\end{array}$ & 5.2 & $\begin{array}{l}\text { new } \beta \text { Cephei star, } \\
\text { V906 Cen }\end{array}$ \\
\hline 62115 & 311884 & $124351-6305.2$ & 10.82 & 11.41452 & 40.3 & 0.16028 & 52.9 & 19.8 & 10.7 & CD Cru (E), W-R star \\
\hline 63489 & 112842 & $130031-6022.5$ & 7.07 & 11.26044 & 8.4 & - & - & - & 7.1 & \\
\hline 67060 & 119608 & $134431-1756.2$ & 7.50 & 10.55676 & 13.8 & - & - & - & 7.9 & \\
\hline 69847 & 124788 & $141742-6047.3$ & 8.98 & 0.45821 & 52.8 & 0.22917 & 70.5 & 97.8 & 5.6 & V999 Cen (E or Ell) \\
\hline & & & & 9.13446 & 21.0 & - & - & - & & \\
\hline 70228 & 125545 & $142204-5817.4$ & 7.42 & 11.50208 & 14.9 & - & - & - & 7.7 & \\
\hline 76642 & 137179 & $153903-8314.0$ & 8.75 & 10.08788 & 13.0 & - & - & - & 4.0 & \\
\hline 80408 & 147421 & $162445-5327.8$ & 9.02 & 5.35481 & 25.1 & 5.35473 & 27.1 & 19.0 & 2.8 & new $\beta$ Cephei star \\
\hline 81216 & 149100 & $163521-5338.9$ & 7.20 & 5.16114 & 6.0 & 5.16118 & 8.1 & 7.7 & 3.8 & new $\beta$ Cephei star \\
\hline 83603 & 154043 & $170519-4704.1$ & 7.08 & 11.17594 & 15.8 & - & - & - & 9.7 & V863 Ara \\
\hline 91822 & 173003 & $184318-0138.7$ & 7.68 & 7.98590 & 9.1 & - & - & - & 3.2 & \\
\hline 98807 & 339483 & $200401+2616.3$ & 8.94 & 3.72410 & 23.4 & 3.72413 & 22.5 & 14.0 & 6.4 & new $\beta$ Cephei star \\
\hline 99327 & 191531 & $200940+2104.7$ & 8.35 & 6.08588 & 26.5 & 6.08589 & 26.0 & 12.2 & 8.6 & $\beta$ Cephei (Handler 2005) \\
\hline
\end{tabular}

equal to 7-8 $M_{\odot}$. Newer calculations (Pamyatnykh 1999) confirmed this and showed in addition that $g$-mode instability extends towards higher masses. The latter instability is confined, however, to the very late main-sequence stages.

This picture of $p$ and $g$ mode instability domains was calculated neglecting the effects of rotation on pulsations. The theory that includes fast rotation is still not fully satisfactory, but it is already clear that rotation changes stability of modes. A good example of the theoretical calculations that include the effect of rotation on the stability of $g$ modes was published by Townsend (2005), who showed that for fast-rotating stars the SPB instability strip widens towards higher masses. The region of possible hybrid behaviour becomes therefore much larger. In addition, the frequencies of modes, especially of non-axisymmetric modes, change considerably. In particular, frequencies of retrograde sectoral mixed modes are shifted to much lower frequencies than they would have in non-rotating stars, i.e., will occur in the range where normally the $g$ modes are observed. On the other hand, frequencies of prograde sectoral $g$ modes shift to the region occupied normally by $p$ modes. It must be also remembered that in the presence of fast rotation modes can no longer be classified in a simple scheme described by three quantum numbers because the geometry of such modes differs from spherical harmonics.

The effects related to fast rotation are impontant not only because some $\beta$ Cephei stars are known to rotate fast. The region of $\beta$ Cephei and SPB stars in the H-R diagram is populated by Be stars which, by definition, rotate very fast. These stars were known to show both short-period variability and line-profile variations. The short-period photometric variability called, after the prototype, the $\lambda$ Eri-type (Balona 1990) was usually attributed to rotationally-induced effects because no clear evidence for multiperiodicity was found (see, e.g., Balona \& James 2002). On the other hand, there were many convincing examples of line-profile variations in Be stars that were interpreted in terms of non-radial pulsations (Baade 1984; Yang et al. 1990; Štefl et al. 1995; Floquet et al. 2000), including multiperiodic behaviour (Rivinius et al. 1998). Despite this, it was not widely accepted that non-radial pulsations are present in Be stars mainly because there was no clear relation between periods seen in photometry and spectroscopy. An excellent review of variabilities observed in Be stars was recently given by Porter \& Rivinius (2003).

A strong argument in favour of non-radial pulsations in $\mathrm{Be}$ stars, as seen in photometry, came from the observations carried out by the MOST satellite, in particular, those of an early Be star, HD 163868, in which Walker et al. (2005b) found over 60 periodicities in three distinct groups. A theoretical interpretation of this variability (Walker et al. 2005b; Dziembowski et al. 2007; Savonije 2007) includes pulsations in $g$ modes. It is therefore reasonable to use the term "SPBe stars" for Be stars with detected $g$-mode pulsations, as proposed by Walker et al. (2005b), although it has to be remembered that many previous investigators already indicated a common cause of periodic variability in SPB and Be stars.

The two grouping of modes seen in HD 163868 around 1.7 and $3.4 \mathrm{~d}^{-1}$ might be a clue to understanding a grouping of modes in $\beta$ Cephei and SPB candidates in the LMC and SMC, found by Kołaczkowski et al. (2004, 2006). In many of these stars, the frequencies of modes grouped around two values whose ratio was close to 2 . Whether fast rotation is responsible for this could be verified by future spectroscopic observations. For the SMC we know that many of these stars are fast-rotating because they show emission in Balmer lines.

Since inclusion of fast rotation enhances the possibility of getting unstable low-frequency modes among early and mid-B stars, we should see them observationally. It seems that we get more and more examples that confirm these predictions. First, 
low-frequency modes were detected in two classical $\beta$ Cephei stars, $v$ Eri (Jerzykiewicz et al. 2005) and 12 Lac (Handler et al. 2006). In the ASAS-3 data (Sect. 3.4), we found five $\beta$ Cephei stars in which low-frequency modes were detected. In addition, another $\beta$ Cephei star with low-frequency modes, HD 133823, was found in Paper I (see also Fig. 19). On the other hand, periodic variations with very short periods were found in some mid and late B-type stars like V2104 Cyg (Uytterhoeven et al. 2007) and stars in NGC 3293 (Handler et al. 2007). A preliminary analysis of the data obtained for the other young open cluster, h Persei (NGC 869) also reveals the presence of mid-B stars, of which some are known as Be stars, showing relatively short periods, even below $0.3 \mathrm{~d}$ (Majewska-Świerzbinowicz et al., in preparation).

The complexity of mode behaviour in the presence of fast rotation may pose a problem as far as the classification of hot pulsators is concerned. This is because period(s) alone may not be sufficient to recognize $\beta$ Cephei and SPB star. Mode identification becomes a neccessity if we want to classify stars pulsating in $p$ modes as $\beta$ Cephei stars, while those pulsating in $g$ modes, as SPB stars.

The other important results that come from the discovery of $103 \beta$ Cephei stars can be summarized as follows:

1. The number of known $\beta$ Cephei stars has grown to over 200 and thus some statistical studies become possible. The sample of new stars is particularly well suited for this purpose because the stars were selected and observed in a homogeneous way.

2. From the new sample, a selection of excellent targets for asteroseismology can be done. These would include stars with large amplitudes (Sect. 3.2), large number of modes, equidistant multiplets (Sect. 3.3) and components of eclipsing binaries (Sect. 3.7).

3. We have found three O-type $\beta$ Cephei stars (Sect. 3.6); this type of pulsation was also found in $\zeta$ Oph from the MOST data (Walker et al. 2005a). Thus, we may conclude that $\beta$ Cephei-type instability extends towards O-type stars, but seems to be confined to very late subtypes (O8-09). This still does not agree well with the theory because calculations do not predict an upper luminosity limit for $p$-mode instability (Pamyatnykh 1999). However, these calculations have not included the effect of stellar wind which may stabilize pulsations for hotter O-type stars.

4. The very recent stability calculations using new opacities (Miglio et al. 2007) indicate a much wider region in the $\mathrm{H}-\mathrm{R}$ diagram where hybrid, $\beta$ Cephei/SPB-type behaviour, should be observed. The inclusion of fast rotation seems to have a similar effect (Townsend 2005). Observationally, this fact is confirmed by the growing number of detections of hybrid stars, including five $\beta$ Cephei stars with long periods we found (Sect. 3.4) as candidates. In order to verify their hybridity, mode identification is needed. A detailed study of these stars may allow testing the physics used in calculations of the evolutionary models of massive stars but also in the theoretical predictions of their pulsational stability.

Finally, it is interesting to note that four stars from our sample, HD 48553, 171305, 173006, and 186610 are located within the "CoRoT eyes" (Baglin et al. 2002) and, if selected for observations by this satellite, could be studied in unprecedented detail.

Acknowledgements. The work was supported by the MNiI/MNiSzW grants No. 1 P03D 01627 and N203 007 31/1328. We greatly acknowledge comments made by Prof. M. Jerzykiewicz and Dr. Gerald Handler, the referee. This research has made use of the SIMBAD database, operated at CDS, Strasbourg, France.

\section{References}

Aerts, C. 2000, A\&A, 361, 245

Aerts, C., Waelkens, C., Daszyńska-Daszkiewicz, J., et al. 2004, A\&A, 415, 241 Baade, D. 1984, A\&A, 135, 101

Baglin, A., Auvergne, M., Catala, C., et al. 2002, in First Eddington Workshop, ed. J. Christensen-Dalsgaard, \& I. Roxburgh, ESA SP-485, 17

Balona, L. A. 1990, MNRAS, 245, 92

Balona, L. A. 1992, MNRAS, 254, 404

Balona, L. A., \& Dziembowski, W. A. 1999, MNRAS, 309, 221

Balona, L. A., \& James, D. J. 2002, MNRAS, 332, 714

Balona, L. A., \& Laney, C. D. 1995, MNRAS, 276, 627

Bruntt, H., \& Buzasi, D. L. 2006, Mem. Soc. Astron. Italiana, 77, 278

Cannon, A. J., \& Pickering, E. C. 1918-1924, The Henry Draper Catalogue, Annals of the Astron. Obs. of Harvard College, Vol. 91-99

Cappa, C. E., \& Benaglia, P. 1998, AJ, 116, 1906

Cuypers, J., Balona, L. A., \& Marang, F. 1989, A\&AS, 81, 151

De Mey, K., Aerts, C., Waelkens, C., \& Van Winckel, H. 1996, A\&A, 310, 164

Deutschmann, W. A., Davis, R. J., \& Schild, R. E. 1976, ApJS, 30, 97

Dziembowski, W. A., \& Pamyatnykh, A. A. 1993, MNRAS, 262, 204

Dziembowski, W. A., Moskalik, P., \& Pamyatnykh, A. A. 1993, MNRAS, 265, 588

Dziembowski, W. A., Daszyńska-Daszkiewicz, J., \& Pamyatnykh, A. A. 2007, MNRAS, 374, 248

Engelbrecht, C. A., \& Balona, L. A. 1986, MNRAS, 219, 449

Feast, M. W., Thackeray, A. D., \& Wesselink, A. J. 1957, MmRAS, 68, 1

Feast, M. W., Stoy, R. H., Thackeray, A. D., \& Wesselink, A. J. 1961, MNRAS, 122,237

FitzGerald, M. P., \& Miller, M. L. 1983, PASP, 95, 361

Floquet, M., Hubert, A. M., Hirata, R., et al. 2000, A\&A, 362, 1020

Freyhammer, L. M., Hensberge, H., Sterken, C., et al. 2005, A\&A, 429, 631

Fullerton, A. W., Gies, D. R., \& Bolton, C. T. 1996, ApJS, 103, 475

Garrison, R. F., Hiltner, W. A., \& Schild, R. E. 1977, ApJS, 35, 111

Handler, G. 2005, IBVS, 5667

Handler, G., Shobbrook, R. R., \& Mokgwetsi, T. 2005, MNRAS, 362, 612

Handler, G., Jerzykiewicz, M., Rodríguez, E., et al. 2006, MNRAS, 365, 327

Handler, G., Tuvikene, T., Lorenz, D., et al. 2007, Comm. Asteroseismol., 150, 193

Herbst, W. 1975, AJ, 80, 212

Hill, P. W. 1970, MNRAS, 150, 23

Hill, P. W., Kilkenny, D., \& van Breda, I. G. 1974, MNRAS, 168, 451

Houk, N. 1978, Michigan Spectral Survey, Ann Arbor, Dep. Astron., Univ. Michigan, 2

Houk, N. 1982, Michigan Spectral Survey, Ann Arbor, Dep. Astron., Univ. Michigan, 3

Houk, N., \& Cowley, A. P. 1975, Michigan Spectral Survey, Ann Arbor, Dep. Astron. Univ. Michigan, 1

Houk, N., \& Smith-Moore, M. 1988, Michigan Spectral Survey, Ann Arbor, Dep. Astron., Univ. Michigan, 4

Houk, N., \& Swift, C. 1999, Michigan Spectral Survey, Ann Arbor, Dep. Astron., Univ. Michigan, 5

Howarth, I. D., \& Prinja, R. K. 1989, ApJS, 69, 527

Howarth, I. D., Siebert, K. W., Hussain, G. A. J., \& Prinja, R. K. 1997, MNRAS, 284,265

Humphreys, R. M. 1978, A\&AS, 38, 309

Jerzykiewicz, M., \& Pamyatnykh, A. A. 2000, PASP, 112, 1341

Jerzykiewicz, M., \& Pigulski, A. 1998, PASPC, 135, 43

Jerzykiewicz, M., \& Sterken, C. 1992, MNRAS, 257, 303

Jerzykiewicz, M., Jarzębowski, T., Musielok, B., \& le Contel, J.-M. 1978, IBVS, 1508

Jerzykiewicz, M., Handler, G., Shobbrook, R. R., et al. 2005, MNRAS, 360, 619

Johansson, K. L. V. 1981, A\&AS, 43, 421

Kaltcheva, N. T. 2003, A\&A, 410, 523

Kaltcheva, N. T., \& Georgiev, L. N. 1994, MNRAS, 269, 289

Kambe, E., Ando, H., \& Hirata, R. 1990, PASJ, 42, 687

Klare, G., \& Neckel, T. 1977, A\&AS, 27, 215

Koen, C. 2001, MNRAS, 321, 44

Koen, C., \& Eyer, L. 2002, MNRAS, 331, 45

Kołaczkowski, Z., Pigulski, A., Soszyński, I., et al. 2004, PASPC, 310, 225

Kołaczkowski, Z., Pigulski, A., Soszyński, I., et al. 2006,

Mem. Soc. Astron. Italiana, 77, 336

Krzesiński, J., \& Pigulski, A. 1997, A\&A, 325, 987

Kuntz, J., \& Stebbins, J. 1916, Pub. Am. Astron. Soc., 3, 272

Lehner, N., Fullerton, A. W., Massa, D., Sembach, K. R., \& Zsargó, J. 2003, ApJ, 589, 526

Levato, H., Morrell, N., Garcia, B., \& Malaroda, S. 1988, ApJS, 68, 319

Lodén, L. O. 1973, A\&AS, 10, 125

Lynn, B. B., Dufton, P. L., Keenan, F. P., et al. 2002, MNRAS, 336, 1287 
MacConnell, D. J. 1981, A\&AS, 44, 387

Mathys, G. 1988, A\&A, 76, 427

McSwain, M. V., \& Gies, D. R. 2005, ApJS, 156, 118

Miglio, A., Montalbán, J., Dupret, M.-A. 2007, MNRAS, 375, L21

Moffat, A. F. J., \& Vogt, N. 1975, A\&AS, 20, 125

Morgan, W. W., Code, A. D., \& Whitford, A. E. 1955, ApJS, 2, 41

Pamyatnykh, A. A. 1999, Acta Astron., 49, 119

Penny, L. R. 1996, ApJ, 463, 737

Penny, L. R., Sprague, A. J., Seago, G., \& Gies, D. R. 2004, ApJ, 617, 1316

Peterson, C. J., \& FitzGerald, M. P. 1988, MNRAS, 235, 1439

Pigulski, A. 2005, Acta Astron., 55, 219

Pigulski, A. 2006, PASPC, 349, 137

Pigulski, A., \& Jerzykiewicz, M. 1988, Acta Astron., 38, 88

Pigulski, A., \& Kołaczkowski, Z. 1998, A\&A, 298, 753

Pigulski, A., \& Pojmański, G. 2008, A\&A, 477, 907 (Paper I)

Pojmański, G. 1997, Acta Astron., 47, 467

Pojmański, G. 2000, Acta Astron., 50, 177

Pojmański, G., Pilecki, B., \& Szczygieł, D. 2005, Acta Astron., 55, 275

Rivinius, T., Baade, D., Štefl, S., et al. 1998, A\&A, 336, 177

Porter, J. M., \& Rivinius, T. 2003, PASP, 115, 1153

Savonije, G. J. 2007, A\&A, 469, 1057

Schild, H. 1985, A\&A, 146, 113

Schmidt-Kaler, T. 1964, ZAp, 58, 217

Shobbrook, R. R., \& Lomb, N. R. 1972, MNRAS, 156, 181
Shobbrook, R. R., Handler, G., Lorenz, D., \& Mogorosi, D. 2006, MNRAS, 369 , 171

Smolec, R., \& Moskalik, P. 2007, MNRAS, 377, 645

Stankov, A., \& Handler, G. 2005, A\&AS, 158, 193

Štefl, S., Baade, D., Harmanec, P., \& Balona, L. A. 1995, A\&A 294, 135

Sterken, C., Snowden, M., Africano, J., et al. 1986, A\&AS, 66, 11

Sterken, C., Pigulski, A., \& Liu, Z. 1993, A\&AS, 98, 383

Townsend, R. H. D. 2005, MNRAS, 360, 465

Uytterhoeven, K., Willems, B., Lefever, K., et al. 2004, A\&A, 427, 581

Uytterhoeven, K., Poretti, E., Rodríguez, E., et al. 2007, A\&A, 470, 1051

Vogt, S. S., \& Penrod, G. D. 1983, ApJ, 275, 661

van den Bergh, S., \& Herbst, W. 1975, AJ, 80, 208

Walborn, N. R. 1973, AJ, 78, 1067

Waelkens, C., \& Lampens, P. 1988, A\&A, 194, 143

Waelkens, C., \& Rufener, F. 1988, A\&A, 201, L5

Waelkens, C., Aerts, C., Kestens, E., Grenon, M., \& Eyer, L. 1998, A\&A,330, 215

Walker, G. A. H. 1963, MNRAS, 125, 141

Walker, G. A. H., Kusching, R., Matthews, J. M., et al. 2005a, ApJ, 623, L145

Walker, G. A. H., Kusching, R., Matthews, J. M., et al. 2005b, ApJ, 635, L77

Wiegert, P., \& Garrison, R. F. 1998, JRASC, 92, 134

Yang, S., Walker, G. A. H., Hill, G. M., \& Harmanec, P. 1990, ApJS, 74, 595

Yudin, R. V. 2001, A\&A, 368, 912 
A. Pigulski and G. Pojmański: $\beta$ Cephei stars in the ASAS-3 data. II., Online Material $p 1$

\section{Online Material}


A. Pigulski and G. Pojmański: $\beta$ Cephei stars in the ASAS-3 data. II., Online Material $p 2$

Table 2. Parameters of the sine-curve fits to the $V$ magnitudes of new $\beta$ Cephei-type stars listed in Table 1. $N_{\text {obs }}$ is the number of observations, the initial epoch $T_{0}$ is equal to 2450000.0 . The other parameters are the following: $T_{\text {max }}^{i}$, time of maximum light for the $i$ th mode, $\sigma_{\text {res }}$, standard deviation of the residuals, DT, detection threshold.

\begin{tabular}{|c|c|c|c|c|c|c|c|}
\hline Star name & Freq. & $N_{\text {obs }}$ & $\begin{array}{c}f_{\mathrm{i}} \\
{\left[\mathrm{d}^{-1}\right]}\end{array}$ & $\begin{array}{c}A_{i} \\
{[\mathrm{mmag}]}\end{array}$ & $\begin{array}{c}T_{\max }^{i}-T_{0} \\
{[\mathrm{~d}]}\end{array}$ & $\begin{array}{c}\sigma_{\text {res }} \\
{[\mathrm{mmag}]}\end{array}$ & $\begin{array}{c}\text { DT } \\
{[\mathrm{mmag}]}\end{array}$ \\
\hline HD 46994 & $f_{1}$ & 322 & $3.90041(4)$ & $7.8(10)$ & $2942.1701(54)$ & 12.8 & 5.1 \\
\hline HD 48553 & $f_{1}$ & 215 & $5.59806(4)$ & $9.1(10)$ & $3143.6502(31)$ & 10.2 & 4.9 \\
\hline HD 67600 & $f_{1}$ & 375 & $3.78661(2)$ & $8.8(08)$ & $2901.9390(35)$ & 10.2 & 3.8 \\
\hline HD 68962 & $f_{1}$ & 191 & $4.76251(6)$ & 7.4(09) & $3069.0758(38)$ & 8.4 & 4.3 \\
\hline HD 69016 & $f_{1}$ & 616 & $5.01186(4)$ & $6.4(09)$ & $2816.8526(46)$ & 15.3 & 4.4 \\
\hline HD 69824 & $f_{1}$ & 351 & $6.06354(4)$ & $5.2(07)$ & $2826.1460(37)$ & 9.5 & 3.6 \\
\hline \multirow[t]{2}{*}{ HD 73568} & $f_{1}$ & 576 & $4.54531(3)$ & $4.8(06)$ & $2865.8111(42)$ & 9.7 & 2.9 \\
\hline & $f_{2}$ & & $6.58022(5)$ & $3.4(06)$ & $2865.6858(41)$ & & \\
\hline \multirow[t]{6}{*}{ HD 74339} & $f_{1}$ & 432 & $5.220103(10)$ & 27.7(09) & $2900.6750(10)$ & 13.1 & 4.5 \\
\hline & $f_{2}$ & & $5.195216(13)$ & 20.7(09) & $2900.7085(14)$ & & \\
\hline & $f_{3}$ & & $5.22221(2)$ & 13.7(09) & $2900.6380(21)$ & & \\
\hline & $f_{4}$ & & $5.23894(3)$ & $11.5(09)$ & $2900.6309(25)$ & & \\
\hline & $f_{5}$ & & $5.20531(3)$ & $10.6(09)$ & $2900.6775(26)$ & & \\
\hline & $f_{6}$ & & $5.25563(3)$ & 10.3(09) & $2900.6234(27)$ & & \\
\hline HD 77769 & $f_{1}$ & 373 & $5.46831(4)$ & $5.5(08)$ & $2849.0965(46)$ & 11.5 & 4.2 \\
\hline HD 86085 & $f_{1}$ & 379 & $7.87529(2)$ & $9.2(08)$ & $2840.1421(17)$ & 10.5 & 3.9 \\
\hline \multirow[t]{6}{*}{ HD 86214} & $f_{1}$ & 512 & $4.442908(13)$ & $14.3(08)$ & 2958.9401(20) & 11.5 & 3.6 \\
\hline & $f_{2}$ & & $4.40462(2)$ & $10.9(07)$ & $2959.0537(24)$ & & \\
\hline & $f_{3}$ & & 4.40967(3) & $8.3(07)$ & $2958.9773(32)$ & & \\
\hline & $f_{4}$ & & $4.41462(3)$ & $9.0(07)$ & 2958.9914(29) & & \\
\hline & $f_{5}$ & & 4.39994(4) & $6.3(08)$ & $2958.8671(42)$ & & \\
\hline & $f_{6}$ & & $4.44618(4)$ & $5.7(08)$ & $2959.0029(48)$ & & \\
\hline HD 86248 & $f_{1}$ & 395 & $8.25590(5)$ & $5.5(08)$ & $2835.6249(29)$ & 11.4 & 4.2 \\
\hline \multirow[t]{5}{*}{ HD 87592} & $f_{1}$ & 790 & $5.15127(2)$ & $6.9(06)$ & $2828.1611(26)$ & 11.3 & 2.8 \\
\hline & $f_{2}$ & & $5.09753(3)$ & $6.8(06)$ & $2828.1263(26)$ & & \\
\hline & $f_{3}$ & & $5.43571(3)$ & $6.3(06)$ & $2828.1232(27)$ & & \\
\hline & $f_{4}$ & & $5.30697(4)$ & $4.1(06)$ & $2828.1605(43)$ & & \\
\hline & $f_{5}$ & & $5.33044(6)$ & $2.9(06)$ & $2828.1757(60)$ & & \\
\hline \multirow[t]{2}{*}{ HD 88844} & $f_{1}$ & 373 & $5.37589(3)$ & $7.5(07)$ & $2881.4701(27)$ & 9.6 & 3.5 \\
\hline & $f_{2}$ & & $6.03797(3)$ & $5.9(07)$ & $2881.4999(31)$ & & \\
\hline HD 90075 & $f_{1}$ & 371 & $4.09526(3)$ & $10.3(09)$ & $2902.7893(34)$ & 12.2 & 4.5 \\
\hline \multirow[t]{4}{*}{ HD 90987} & $f_{1}$ & 368 & $4.702781(07)$ & $39.6(10)$ & $2873.8688(08)$ & 13.2 & 4.9 \\
\hline & $f_{2}$ & & $4.56652(2)$ & $16.1(10)$ & $2874.0208(21)$ & & \\
\hline & $f_{3}$ & & $4.59644(3)$ & $8.7(10)$ & $2873.8866(39)$ & & \\
\hline & $f_{4}$ & & $4.36623(4)$ & $6.7(10)$ & $2873.9638(54)$ & & \\
\hline \multirow[t]{2}{*}{ HD 91651} & $f_{1}$ & 525 & $7.38876(3)$ & $5.6(06)$ & $2876.1314(23)$ & 9.7 & 3.0 \\
\hline & $f_{2}$ & & $7.10851(5)$ & $3.5(06)$ & $2876.1737(38)$ & & \\
\hline \multirow[t]{5}{*}{ HD 92291} & $f_{1}$ & 385 & $5.528817(10)$ & 23.3(09) & $2884.2608(11)$ & 11.8 & 4.2 \\
\hline & $f_{2}$ & & $5.454284(13)$ & $17.5(09)$ & $2884.2939(15)$ & & \\
\hline & $f_{3}$ & & $5.90508(3)$ & $8.9(09)$ & $2884.3169(26)$ & & \\
\hline & $f_{4}$ & & $5.46980(3)$ & 7.4(09) & $2884.2436(34)$ & & \\
\hline & $f_{5}$ & & $5.72446(4)$ & $6.2(08)$ & $2884.2426(40)$ & & \\
\hline HD 93113 & $f_{1}$ & 690 & $5.57541(5)$ & $3.7(06)$ & 2856.9991(46) & 11.2 & 3.1 \\
\hline HD 93341 & $f_{1}$ & 691 & $5.08749(3)$ & $8.2(07)$ & $2881.2753(24)$ & 12.1 & 3.3 \\
\hline \multirow[t]{5}{*}{ HD 94065} & $f_{1}$ & 364 & $4.810101(10)$ & $20.3(08)$ & $2861.4725(13)$ & 10.3 & 3.8 \\
\hline & $f_{2}$ & & $5.02213(3)$ & $11.1(08)$ & $2861.4386(24)$ & & \\
\hline & $f_{3}$ & & $5.01919(2)$ & $13.5(08)$ & $2861.5756(19)$ & & \\
\hline & $f_{4}$ & & $5.48660(3)$ & $9.3(08)$ & $2861.4498(24)$ & & \\
\hline & $f_{5}$ & & $5.02074(3)$ & $8.5(08)$ & $2861.4767(30)$ & & \\
\hline \multirow[t]{3}{*}{ HD 94345} & $f_{1}$ & 487 & $5.16834(2)$ & $9.4(07)$ & $2994.2366(23)$ & 10.6 & 3.4 \\
\hline & $f_{2}$ & & $5.17063(4)$ & $5.4(07)$ & $2994.3449(39)$ & & \\
\hline & $f_{3}$ & & $5.57486(5)$ & $3.8(07)$ & $2994.2818(53)$ & & \\
\hline HD 94900 & $f_{1}$ & 468 & $4.842766(11)$ & $16.8(08)$ & $2930.6143(14)$ & 11.2 & 3.7 \\
\hline \multirow[t]{2}{*}{ HD 95568} & $f_{1}$ & 365 & $6.152355(15)$ & $16.9(10)$ & $2857.4896(15)$ & 12.7 & 4.7 \\
\hline & $f_{2}$ & & $6.13549(3)$ & $10.4(10)$ & $2857.4369(24)$ & & \\
\hline HD 96882 & $f_{1}$ & 355 & $6.22739(4)$ & $6.6(09)$ & $2909.9008(33)$ & 11.3 & 4.3 \\
\hline \multirow[t]{2}{*}{ HD 96901} & $f_{1}$ & 340 & $4.163209(11)$ & $19.2(08)$ & $2900.2036(16)$ & 10.1 & 3.9 \\
\hline & $f_{2}$ & & $4.128028(17)$ & $11.4(08)$ & $2900.2624(27)$ & & \\
\hline HD 97629 & $f_{1}$ & 366 & $5.178579(17)$ & $13.3(08)$ & 2920.9323(19) & 11.0 & 4.1 \\
\hline
\end{tabular}


Table 2. continued.

\begin{tabular}{|c|c|c|c|c|c|c|c|}
\hline Star name & Freq. & $N_{\text {obs }}$ & $\begin{array}{c}\begin{array}{c}f_{\mathrm{i}} \\
{\left[\mathrm{d}^{-1}\right]}\end{array} \\
\end{array}$ & $\begin{array}{c}A_{i} \\
{[\mathrm{mmag}]}\end{array}$ & $\begin{array}{c}\overline{T_{\max }^{i}-T_{0}} \\
{[\mathrm{~d}]}\end{array}$ & $\begin{array}{c}\sigma_{\text {res }} \\
{[\mathrm{mmag}]}\end{array}$ & $\begin{array}{c}\text { DT } \\
{[\mathrm{mmag}]}\end{array}$ \\
\hline \multirow[t]{3}{*}{ HD 98260} & $\begin{array}{l}1 \\
f_{1}\end{array}$ & 350 & $4.514510(19)$ & $14.2(09)$ & $2896.2087(23)$ & 12.0 & 4.6 \\
\hline & $f_{2}$ & & $4.44349(3)$ & $9.8(09)$ & $2896.1907(34)$ & & \\
\hline & $f_{3}$ & & $4.48065(3)$ & $10.3(09)$ & $2896.0648(31)$ & & \\
\hline HD 99024 & $f_{1}$ & 406 & $7.03125(4)$ & $7.0(08)$ & $2992.2193(28)$ & 12.0 & 4.2 \\
\hline \multirow[t]{2}{*}{ HD 99205} & $f_{1}$ & 645 & $3.822544(10)$ & $18.1(07)$ & $2882.5932(16)$ & 12.3 & 3.5 \\
\hline & $f_{2}$ & & $1.24684(4)$ & $5.7(07)$ & $2882.334(16)$ & & \\
\hline \multirow{3}{*}{ HD 100355} & $f_{1}$ & 670 & 7.507394(15) & $10.3(06)$ & $2865.7310(13)$ & 10.9 & 3.0 \\
\hline & $f_{2}$ & & $7.112278(16)$ & $10.2(06)$ & $2865.7452(13)$ & & \\
\hline & $f_{3}$ & & $6.93375(4)$ & $4.2(06)$ & $2865.6535(32)$ & & \\
\hline \multirow[t]{2}{*}{ HD 101794} & $f_{1}$ & 311 & $4.45140(3)$ & $14.6(13)$ & $2737.1557(31)$ & 15.4 & 6.2 \\
\hline & $f_{2}$ & & $1.83951(4)$ & $11.2(13)$ & $2736.998(10)$ & & \\
\hline HD 101838 & $f_{1}$ & 352 & $3.12760(3)$ & $10.3(09)$ & $2865.5247(45)$ & 11.8 & 4.9 \\
\hline \multirow[t]{2}{*}{ HD 102505} & $f_{1}$ & 332 & $4.750770(15)$ & 13.7(08) & 2865.3997(19) & 9.9 & 3.9 \\
\hline & $f_{2}$ & & $4.52947(4)$ & $5.8(08)$ & $2865.4186(47)$ & & \\
\hline \multirow[t]{3}{*}{ HD 103007} & $f_{1}$ & 346 & $5.707239(18)$ & $12.2(09)$ & $2849.9655(20)$ & 11.2 & 4.2 \\
\hline & $f_{2}$ & & $5.75454(3)$ & $10.5(09)$ & $2849.9456(23)$ & & \\
\hline & $f_{3}$ & & $5.67467(3)$ & $9.9(09)$ & $2849.9219(25)$ & & \\
\hline HD 103320 & $f_{1}$ & 351 & $4.71222(2)$ & $8.0(08)$ & $2894.0787(32)$ & 9.8 & 3.7 \\
\hline HD 10 & $f_{1}$ & 330 & $5.582626(18)$ & $12.2(09)$ & $2859.0861(20)$ & 10.9 & 4.3 \\
\hline HD 104257 & $f_{1}$ & 321 & $6.868277(11)$ & $20.6(09)$ & $2852.5829(10)$ & 11.0 & 4.4 \\
\hline \multirow{2}{*}{ HD 104465} & $f_{1}$ & 330 & $5.518702(17)$ & $14.8(10)$ & $2862.6886(19)$ & 12.2 & 4.8 \\
\hline & $f_{2}$ & & 5.395199(19) & $14.2(10)$ & $2862.7541(20)$ & & \\
\hline HD 104795 & $f_{1}$ & 330 & $6.23215(4)$ & $5.3(07)$ & $2883.9586(31)$ & 8.4 & 3.3 \\
\hline HD 106345 & $f_{1}$ & 440 & $6.612477(12)$ & $15.0(07)$ & $2843.0066(11)$ & 10.2 & 3.4 \\
\hline HD 108628 & $f_{1}$ & 637 & $6.74262(4)$ & $4.9(06)$ & $2867.9268(30)$ & 10.9 & 3.1 \\
\hline HD 108769 & $f_{1}$ & 317 & $7.54085(4)$ & $5.4(08)$ & $2816.7267(32)$ & 10.1 & 4.0 \\
\hline HD 110498 & $f_{1}$ & 325 & $4.15383(2)$ & $10.3(09)$ & $2875.1748(30)$ & 10.5 & 4.1 \\
\hline HD 111377 & $f_{1}$ & 342 & $8.83122(4)$ & $6.4(09)$ & $2933.8111(25)$ & 11.4 & 4.4 \\
\hline HD 111578 & $f_{1}$ & 344 & $4.71676(3)$ & $8.8(09)$ & $2914.2855(34)$ & 11.7 & 4.5 \\
\hline \multirow[t]{2}{*}{ HD 113013} & $f_{1}$ & 393 & $6.47119(3)$ & $9.5(08)$ & $2986.8716(22)$ & 11.7 & 4.2 \\
\hline & $f_{2}$ & & $6.26890(4)$ & $6.4(08)$ & $2986.8920(34)$ & & \\
\hline \multirow[t]{2}{*}{ HD 114444} & $f_{1}$ & 508 & $4.81618(4)$ & $8.0(10)$ & $2709.0849(43)$ & 16.3 & 5.1 \\
\hline & $f_{2}$ & & $5.11817(5)$ & $6.6(10)$ & $2709.1424(49)$ & & \\
\hline HD 1 & $f_{1}$ & 620 & $7.24916(3)$ & $8.3(07)$ & $2854.8118(19)$ & 12.4 & 3.5 \\
\hline HD 11 & $f_{1}$ & 330 & $14.57715(3)$ & $11.8(11)$ & $2869.9716(10)$ & 14.0 & 5.5 \\
\hline HD 116538 & $f_{1}$ & 365 & $4.52246(3)$ & $10.6(11)$ & $2859.5868(36)$ & 14.4 & 5.4 \\
\hline \multirow[t]{2}{*}{ HD 116827} & $f_{1}$ & 555 & $2.21158(3)$ & $12.5(09)$ & $2968.2039(52)$ & 15.0 & 4.5 \\
\hline & $f_{2}$ & & $4.45852(3)$ & $10.4(09)$ & $2967.9669(31)$ & & \\
\hline \multirow[t]{2}{*}{ HD 117357} & $f_{1}$ & 561 & $2.08106(3)$ & $8.8(07)$ & $2967.5187(57)$ & 11.0 & 3.3 \\
\hline & $f_{2}$ & & $6.53990(4)$ & $6.2(07)$ & $2967.7211(26)$ & & \\
\hline HD 117687 & $f_{1}$ & 515 & $6.17482(3)$ & $6.8(07)$ & $2944.0268(25)$ & 10.6 & 3.3 \\
\hline HD 117704 & $f_{1}$ & 559 & $4.65537(3)$ & $8.4(08)$ & 2971.8997(34) & 13.8 & 4.2 \\
\hline HD 119252 & $f_{1}$ & 326 & $8.78623(3)$ & $13.3(11)$ & $2863.9353(14)$ & 13.5 & 5.3 \\
\hline \multirow[t]{2}{*}{ HD 119910} & $f_{1}$ & 314 & $7.03488(5)$ & $6.9(07)$ & $3161.7560(24)$ & 9.2 & 3.7 \\
\hline & $f_{2}$ & & $5.95546(6)$ & $4.6(07)$ & $3161.7249(43)$ & & \\
\hline \multirow[t]{2}{*}{ HD 122831} & $f_{1}$ & 462 & $5.008025(17)$ & $14.9(09)$ & $3023.4327(19)$ & 12.7 & 4.1 \\
\hline & $f_{2}$ & & 1.8 & $4.9(08)$ & $3023.337(15)$ & & \\
\hline \multirow[t]{4}{*}{ HD 123077} & $f_{1}$ & 444 & $7.043592(14)$ & $23.6(11)$ & $2811.6284(10)$ & 15.7 & 5.3 \\
\hline & $f_{2}$ & & $6.95371(3)$ & $10.2(10)$ & $2811.6703(25)$ & & \\
\hline & $f_{3}$ & & $6.62910(4)$ & $9.8(11)$ & $2811.5793(27)$ & & \\
\hline & $f_{4}$ & & 7.70277(6) & $6.4(11)$ & $2811.5844(35)$ & & \\
\hline HD 126357 & $f_{1}$ & 516 & $4.06927(3)$ & $8.4(09)$ & $2754.2463(38)$ & 13.1 & 4.1 \\
\hline & $f_{2}$ & & $8.54271(4)$ & $8.1(08)$ & $2754.1686(19)$ & & \\
\hline & $f_{3}$ & & $8.07010(4)$ & $7.8(08)$ & $2754.0913(21)$ & & \\
\hline HD 131805 & $f_{1}$ & 546 & 7.38299(4) & $5.9(08)$ & $2808.2366(28)$ & 12.8 & 3.9 \\
\hline HD 132320 & $f_{1}$ & 377 & $8.44635(3)$ & $7.6(09)$ & $2808.8232(22)$ & 12.1 & 4.4 \\
\hline HD 137405 & $f_{1}$ & 677 & $4.334276(15)$ & $11.3(06)$ & 2924.5163(19) & 10.9 & 2.9 \\
\hline HD 142754 & $f_{1}$ & 289 & $8.51115(5)$ & $6.8(10)$ & $2880.1249(28)$ & 11.9 & 5.0 \\
\hline HD 145537 & $f_{1}$ & 284 & $5.481110(17)$ & $22.9(13)$ & 2849.0793(17) & 15.3 & 6.5 \\
\hline & $f_{2}$ & & $5.18768(3)$ & $16.4(13)$ & $2849.0567(24)$ & & \\
\hline HD 146442 & $f_{1}$ & 328 & $6.57248(3)$ & $9.1(09)$ & $2899.1253(22)$ & 10.7 & 4.3 \\
\hline & $f_{2}$ & & $6.76650(4)$ & $6.0(09)$ & $2899.0557(33)$ & & \\
\hline
\end{tabular}


Table 2. continued.

\begin{tabular}{|c|c|c|c|c|c|c|c|}
\hline Star name & Freq. & $N_{\text {obs }}$ & $\begin{array}{c}f_{\mathrm{i}} \\
{\left[\mathrm{d}^{-1}\right]}\end{array}$ & $\begin{array}{c}A_{i} \\
{[\mathrm{mmag}]}\end{array}$ & $\begin{array}{c}T_{\max }^{i}-T_{0} \\
\text { [d] }\end{array}$ & $\begin{array}{c}\sigma_{\text {res }} \\
{[\mathrm{mmag}]}\end{array}$ & $\begin{array}{c}\text { DT } \\
{[\mathrm{mmag}]}\end{array}$ \\
\hline & $f_{3}$ & & $5.50073(5)$ & $5.4(08)$ & $2899.0582(46)$ & & \\
\hline HD 147421 & $f_{1}$ & 642 & $5.354726(06)$ & $27.1(06)$ & $2899.7481(06)$ & 9.9 & 2.8 \\
\hline HD 149100 & $f_{1}$ & 284 & $5.16118(4)$ & $8.1(08)$ & $3128.9118(30)$ & 9.2 & 3.8 \\
\hline \multirow[t]{2}{*}{ HD 150927} & $f_{1}$ & 328 & $4.834676(15)$ & $24.7(11)$ & $2916.1050(16)$ & 14.5 & 5.7 \\
\hline & $f_{2}$ & & $4.854070(16)$ & $22.4(12)$ & $2916.1120(17)$ & & \\
\hline HD 151158 & $f_{1}$ & 307 & $5.501130(17)$ & $17.5(10)$ & 2892.7554(17) & 12.4 & 5.3 \\
\hline \multirow[t]{2}{*}{ HD 152060} & $f_{1}$ & 303 & $9.57323(3)$ & $8.5(08)$ & $2884.4547(17)$ & 10.3 & 4.2 \\
\hline & $f_{2}$ & & $12.19261(3)$ & $8.8(08)$ & $2884.4692(12)$ & & \\
\hline \multirow[t]{2}{*}{ HD 152162} & $f_{1}$ & 490 & $6.031971(06)$ & $33.1(06)$ & $2867.8221(05)$ & 9.7 & 3.3 \\
\hline & $f_{2}$ & & $6.02169(2)$ & $8.6(06)$ & 2867.7874(19) & & \\
\hline \multirow[t]{3}{*}{ HD 152372} & $f_{1}$ & 479 & $8.64280(3)$ & $8.6(07)$ & $2840.2601(15)$ & 10.6 & 3.5 \\
\hline & $f_{2}$ & & $8.33283(4)$ & $5.7(07)$ & $2840.2841(23)$ & & \\
\hline & $f_{3}$ & & $8.68004(5)$ & 4.4(07) & $2840.3162(28)$ & & \\
\hline HD 153772 & $f_{1}$ & 351 & $3.275788(10)$ & $23.9(08)$ & $2874.3683(16)$ & 10.8 & 4.1 \\
\hline \multirow[t]{2}{*}{ HD 154500} & $f_{1}$ & 911 & $6.98730(3)$ & $8.6(06)$ & 2991.7359(17) & 13.1 & 3.6 \\
\hline & $f_{2}$ & & 7.05945(3) & $7.9(06)$ & 2991.8199(18) & & \\
\hline HD 155407 & $f_{1}$ & 319 & $5.552652(09)$ & $28.9(09)$ & $2865.0140(09)$ & 11.2 & 4.4 \\
\hline HD 156172 & $f_{1}$ & 294 & $6.97258(3)$ & $10.8(10)$ & $2909.6293(21)$ & 12.4 & 5.1 \\
\hline HD 156321 & $f_{1}$ & 631 & $8.72485(5)$ & $4.0(05)$ & $3098.6986(22)$ & 8.3 & 2.5 \\
\hline \multirow{3}{*}{ HD 159792} & $f_{1}$ & 536 & $7.000049(10)$ & 25.7(09) & $2801.4000(08)$ & 14.7 & 4.5 \\
\hline & $f_{2}$ & & $7.24311(3)$ & 8.4(09) & $2801.3910(24)$ & & \\
\hline & $f_{3}$ & & $6.91847(5)$ & $5.6(09)$ & $2801.3297(37)$ & & \\
\hline \multirow[t]{4}{*}{ HD 161633} & $f_{1}$ & 332 & $6.10395(2)$ & $12.8(10)$ & $2828.7628(21)$ & 12.9 & 5.0 \\
\hline & $f_{2}$ & & $6.26392(3)$ & $11.4(10)$ & $2828.6801(23)$ & & \\
\hline & $f_{3}$ & & $5.90006(3)$ & $10.6(10)$ & $2828.6718(26)$ & & \\
\hline & $f_{4}$ & & $5.60499(5)$ & $5.9(10)$ & 2828.7392(49) & & \\
\hline HD 164188 & $f_{1}$ & 413 & $5.71220(4)$ & $5.8(07)$ & 2903.4761(30) & 9.3 & 3.3 \\
\hline \multirow[t]{5}{*}{ HD 164741} & $f_{1}$ & 474 & $5.119632(19)$ & $13.9(08)$ & 2846.7981(19) & 12.0 & 3.9 \\
\hline & $f_{2}$ & & $5.16521(2)$ & $12.0(08)$ & $2846.8063(20)$ & & \\
\hline & $f_{3}$ & & $5.56809(3)$ & $8.6(08)$ & $2846.8316(27)$ & & \\
\hline & $f_{4}$ & & $5.12946(4)$ & $6.6(08)$ & $2846.6758(38)$ & & \\
\hline & $f_{5}$ & & $4.96110(5)$ & $5.1(08)$ & $2846.7909(51)$ & & \\
\hline \multirow[t]{2}{*}{ HD 165955} & $f_{1}$ & 762 & $11.906129(19)$ & 11.0(06) & $3383.4615(07)$ & 10.9 & 3.2 \\
\hline & $f_{2}$ & & $10.16581(4)$ & 7.4(06) & $3383.4489(12)$ & & \\
\hline \multirow[t]{3}{*}{ HD 166304} & $f_{1}$ & 633 & $7.03862(3)$ & $5.9(06)$ & $2888.9596(20)$ & 9.3 & 2.7 \\
\hline & $f_{2}$ & & $6.33937(6)$ & $3.3(06)$ & $2888.9669(39)$ & & \\
\hline & $f_{3}$ & & $7.55353(6)$ & $3.3(06)$ & $2888.8784(33)$ & & \\
\hline \multirow[t]{4}{*}{ HD 167003} & $f_{1}$ & 758 & $6.77267(3)$ & $10.5(06)$ & $3395.4748(13)$ & 10.8 & 3.2 \\
\hline & $f_{2}$ & & $7.54580(4)$ & $6.8(06)$ & $3395.5124(18)$ & & \\
\hline & $f_{3}$ & & $7.01603(4)$ & $6.2(06)$ & $3395.4349(21)$ & & \\
\hline & $f_{4}$ & & $5.37835(4)$ & $5.7(06)$ & $3395.5481(29)$ & & \\
\hline HD 167451 & $f_{1}$ & 330 & $4.37474(3)$ & 11.5(09) & $2849.3065(29)$ & 11.2 & 4.4 \\
\hline \multirow[t]{2}{*}{ HD 168015} & $f_{1}$ & 349 & $6.324050(17)$ & $12.4(07)$ & $2830.9715(14)$ & 9.0 & 3.4 \\
\hline & $f_{2}$ & & $5.07961(2)$ & $10.1(07)$ & $2831.0446(21)$ & & \\
\hline \multirow[t]{2}{*}{ HD 168050} & $f_{1}$ & 698 & $5.5486 \ldots[\mathrm{var}]$ & $27.7(10)$ & see Table 5 & 12.2 & 3.7 \\
\hline & $f_{2}$ & & $5.251039(15)$ & $20.7(10)$ & 2921.1561(15) & & \\
\hline HD 168675 & $f_{1}$ & 596 & $6.35906(2)$ & $8.7(06)$ & 2867.6437(17) & 9.7 & 2.8 \\
\hline & $f_{2}$ & & $4.18134(4)$ & $5.1(06)$ & $2867.6660(42)$ & & \\
\hline HD 168750 & $f_{1}$ & 547 & $4.061855(12)$ & $15.5(06)$ & 2849.0681(16) & 10.2 & 3.1 \\
\hline & $f_{2}$ & & $4.025154(19)$ & $9.5(06)$ & $2849.1496(26)$ & & \\
\hline & $f_{3}$ & & $4.04348(4)$ & $5.1(06)$ & 2849.2132(49) & & \\
\hline HD 169173 & $f_{1}$ & 798 & $5.31303(3)$ & $6.7(07)$ & $2883.5407(29)$ & 13.0 & 3.6 \\
\hline HD 169601 & $f_{1}$ & 612 & $5.173682(11)$ & $23.0(08)$ & $2844.3032(10)$ & 12.3 & 3.9 \\
\hline & $f_{2}$ & & $5.231036(18)$ & $14.2(07)$ & $2844.3146(16)$ & & \\
\hline & $f_{3}$ & & $5.08657(3)$ & $8.2(07)$ & $2844.2888(28)$ & & \\
\hline HD 171141 & $f_{1}$ & 349 & $11.19358(4)$ & 7.1(07) & $2845.9130(14)$ & 9.0 & 3.5 \\
\hline HD 171305 & $f_{1}$ & 288 & $5.15860(2)$ & $10.0(08)$ & $2937.3750(24)$ & 9.3 & 3.9 \\
\hline HD 171344 & $f_{1}$ & 316 & $5.41534(5)$ & $7.5(10)$ & $2828.6084(37)$ & 11.8 & 4.7 \\
\hline HD 172140 & $f_{1}$ & 1346 & $6.61531(3)$ & $8.8(04)$ & $2867.4248(11)$ & 9.5 & 2.1 \\
\hline & $f_{2}$ & & $6.29880(3)$ & $7.8(04)$ & 2867.3434(14) & & \\
\hline & $f_{3}$ & & $7.17605(4)$ & $6.2(04)$ & $2867.4152(14)$ & & \\
\hline & $f_{4}$ & & $6.25128(6)$ & $4.2(04)$ & $2867.4350(25)$ & & \\
\hline & $f_{5}$ & & 7.56117(9) & $2.6(04)$ & $2867.4188(30)$ & & \\
\hline HD 172427 & $f_{1}$ & 283 & $4.01969(3)$ & $10.6(09)$ & $2840.9159(32)$ & 9.9 & 4.2 \\
\hline
\end{tabular}


A. Pigulski and G. Pojmański: $\beta$ Cephei stars in the ASAS-3 data. II., Online Material $p 5$

Table 2. continued.

\begin{tabular}{rcclrrrr}
\hline \hline Star name & Freq. & $N_{\text {obs }}$ & \multicolumn{1}{c}{$\begin{array}{c}f_{\mathrm{i}} \\
{\left[\mathrm{d}^{-1}\right]}\end{array}$} & $\begin{array}{c}A_{i} \\
{[\mathrm{mmag}]}\end{array}$ & $\begin{array}{c}T_{\max }^{i}-T_{0} \\
{[\mathrm{~d}]}\end{array}$ & $\begin{array}{c}\sigma_{\text {res }} \\
{[\mathrm{mmag}]}\end{array}$ & $\begin{array}{c}\text { DT } \\
{[\mathrm{mmag}]}\end{array}$ \\
\hline HD 173006 & $f_{1}$ & 269 & $5.878262(06)$ & $87.8(18)$ & $2892.8486(05)$ & 20.7 & 9.0 \\
& $2 f_{1}$ & & 11.756524 & $22.9(18)$ & $2892.7824(11)$ & & \\
HD 173502 & $f_{1}$ & 1342 & $5.496527(07)$ & $37.3(04)$ & $2865.1104(04)$ & 9.5 & 2.2 \\
& $f_{2}$ & & $5.540236(15)$ & $16.4(04)$ & $2865.0533(07)$ & & \\
& $f_{3}$ & & $5.33345(3)$ & $8.1(04)$ & $2865.0716(15)$ & & \\
HD 178987 & $f_{4}$ & & $5.15802(8)$ & $3.2(04)$ & $2865.0158(37)$ & & \\
HD 180032 & $f_{1}$ & 339 & $7.14660(3)$ & $10.5(11)$ & $2854.0577(23)$ & 14.4 & 5.7 \\
& $f_{1}$ & 287 & $6.86806(3)$ & $8.6(08)$ & $2820.2828(21)$ & 9.3 & 3.9 \\
HD 186610 & $f_{2}$ & & $7.18637(4)$ & $5.9(08)$ & $2820.4152(29)$ & & \\
& $f_{1}$ & 726 & $5.499983(17)$ & $15.6(06)$ & $3023.3878(11)$ & 10.8 & 2.9 \\
& $f_{2}$ & & $5.76342(3)$ & $13.0(06)$ & $3023.3979(12)$ & & \\
& $f_{3}$ & & $5.46127(3)$ & $11.4(06)$ & $3023.4022(15)$ & & \\
HD 187536 & $f_{4}$ & & $5.53861(5)$ & $5.3(06)$ & $3023.5457(31)$ & & \\
& $f_{1}$ & 285 & $4.58572(4)$ & $6.7(09)$ & $2796.7750(46)$ & 10.7 & 4.5 \\
\hline
\end{tabular}


A. Pigulski and G. Pojmański: $\beta$ Cephei stars in the ASAS-3 data. II., Online Material $p 6$
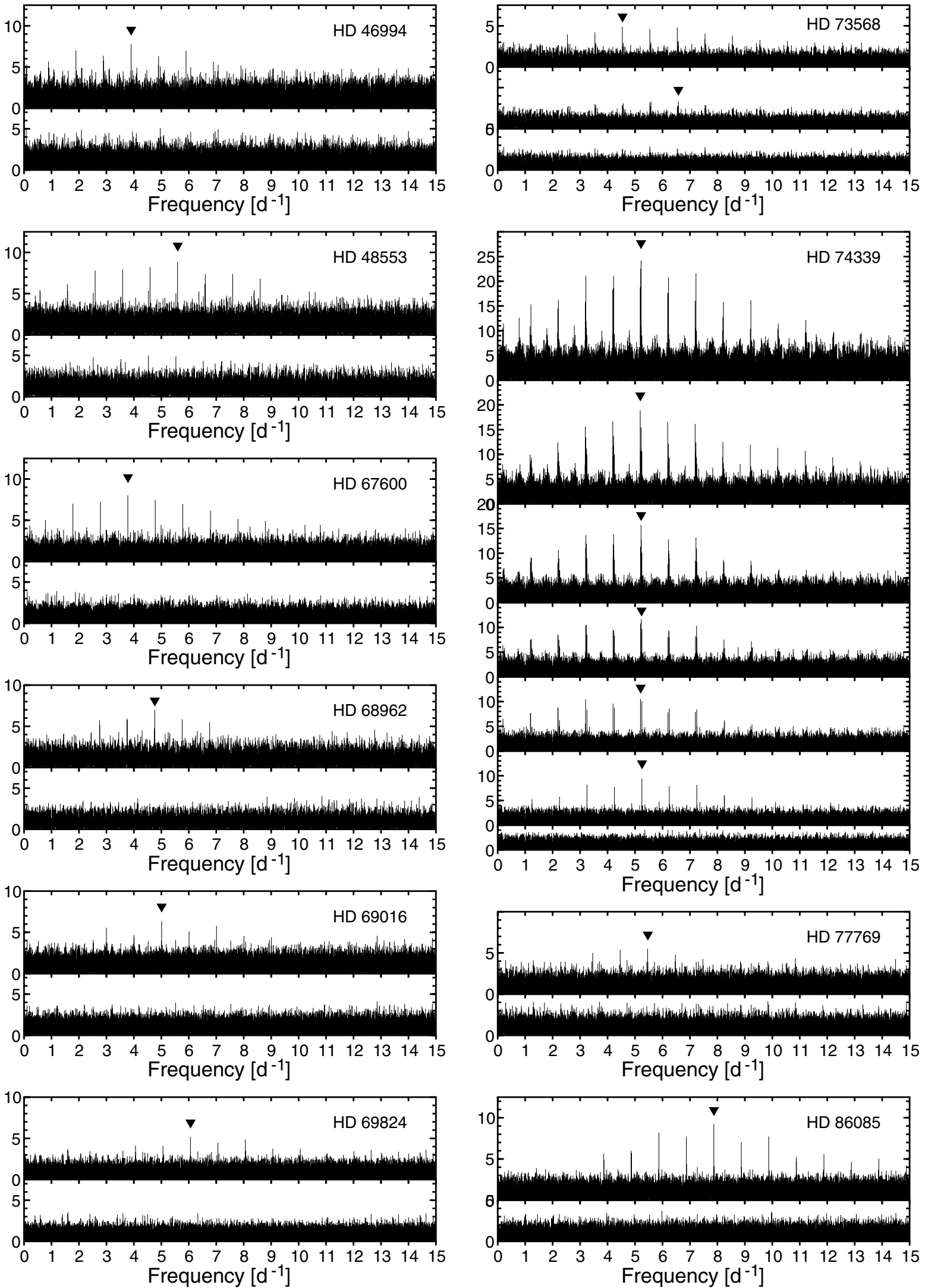

Fig. 2. Fourier periodograms of the ASAS-3 data of ten $\beta$ Cephei-type stars discussed in the paper: HD 46994, 48553, 67600, 68962, 69016, $69824,73568,74339,77769$, and 86085. Lower panels show consecutive steps of prewhitening. Frequencies of the detected modes are indicated by inverted triangles. Ordinate is the amplitude expressed in mmag. 
A. Pigulski and G. Pojmański: $\beta$ Cephei stars in the ASAS-3 data. II., Online Material $p 7$
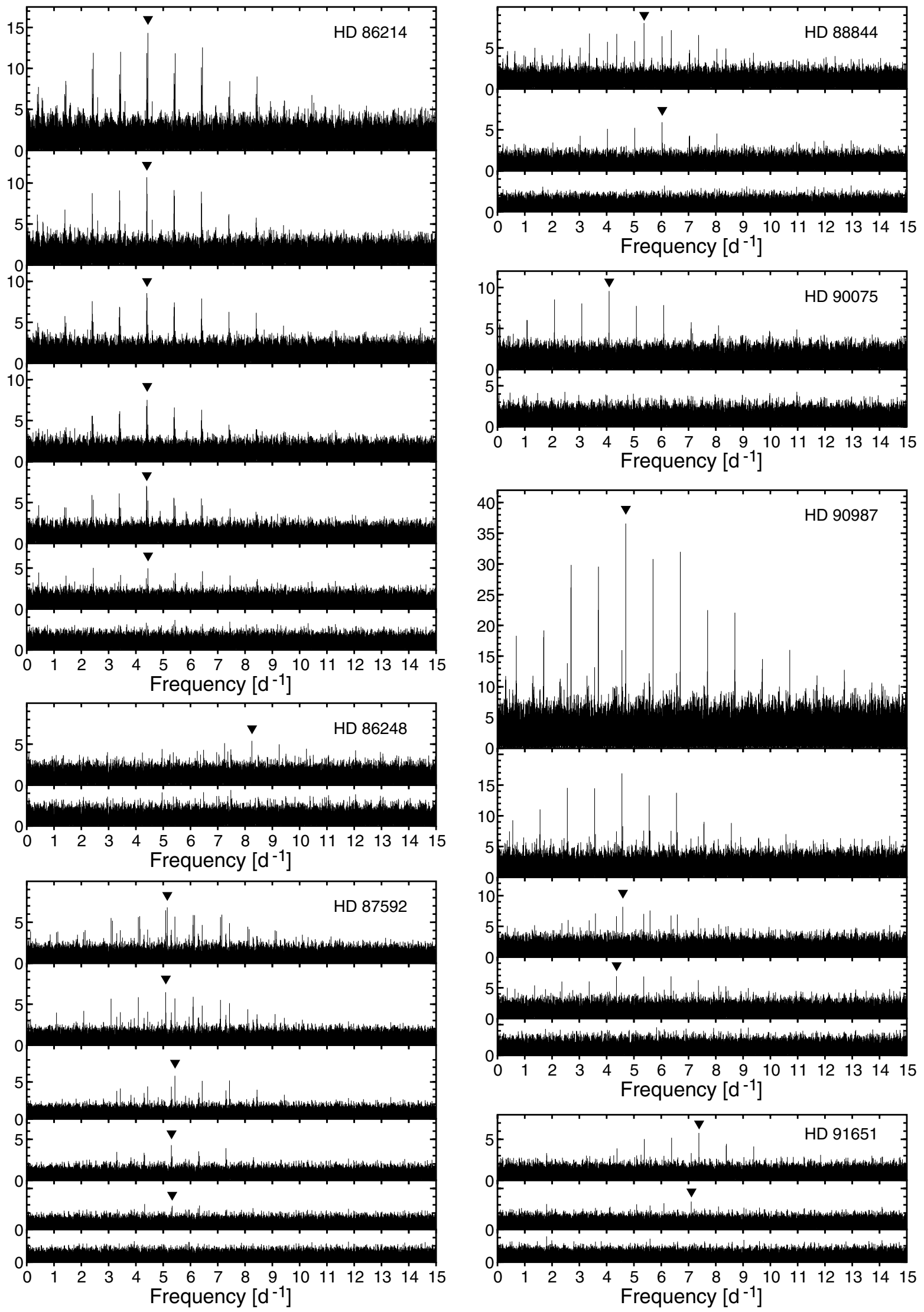

Fig. 3. The same as in Fig. 2, but for HD 86214, 86248, 87592, 88844, 90075, 90987, and 91651. 
A. Pigulski and G. Pojmański: $\beta$ Cephei stars in the ASAS-3 data. II., Online Material $p 8$
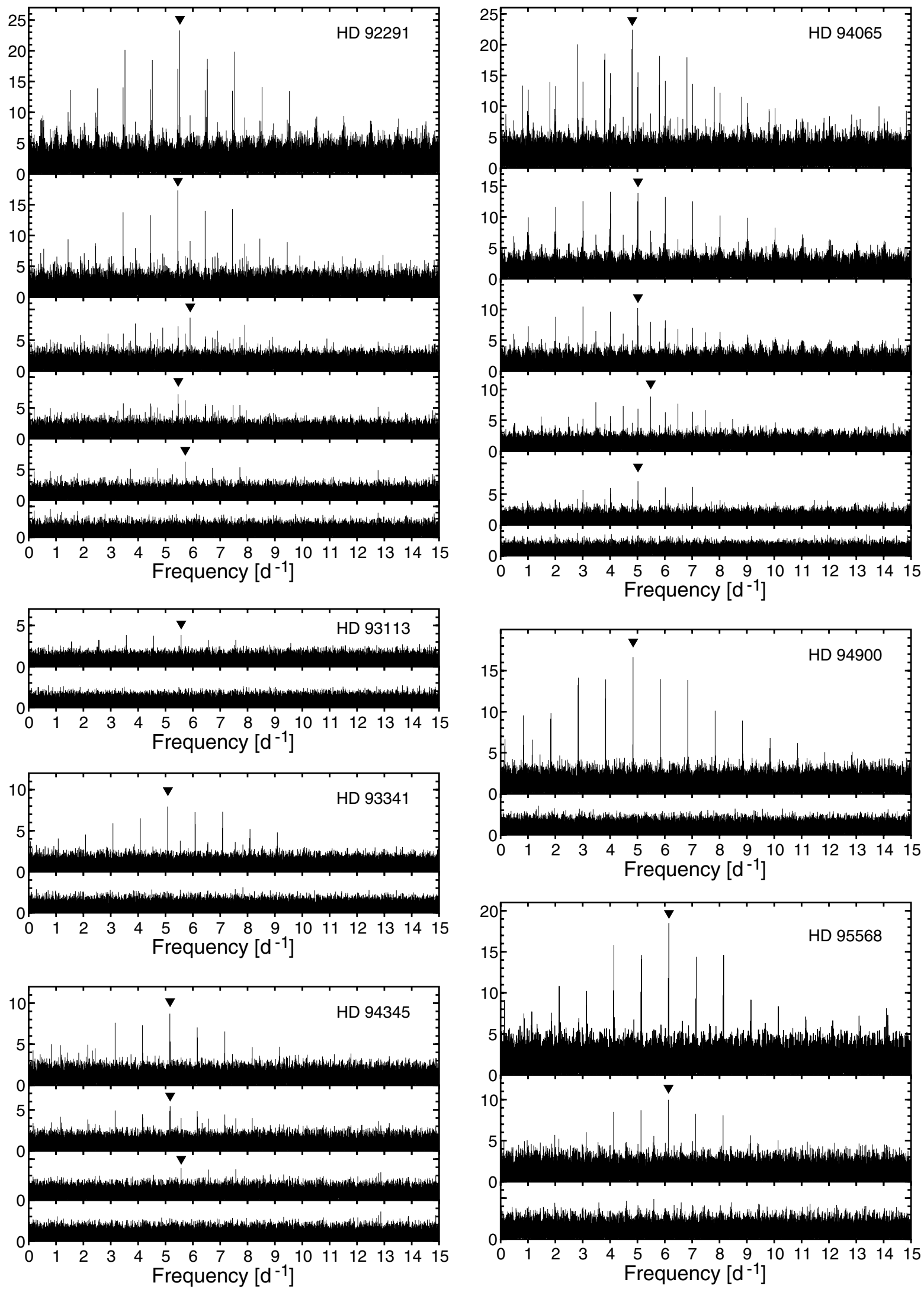

Fig. 4. The same as in Fig. 2, but for HD 92291, 93113, 93341, 94065, 94345, 94900, and 95568. 
A. Pigulski and G. Pojmański: $\beta$ Cephei stars in the ASAS-3 data. II., Online Material $p 9$
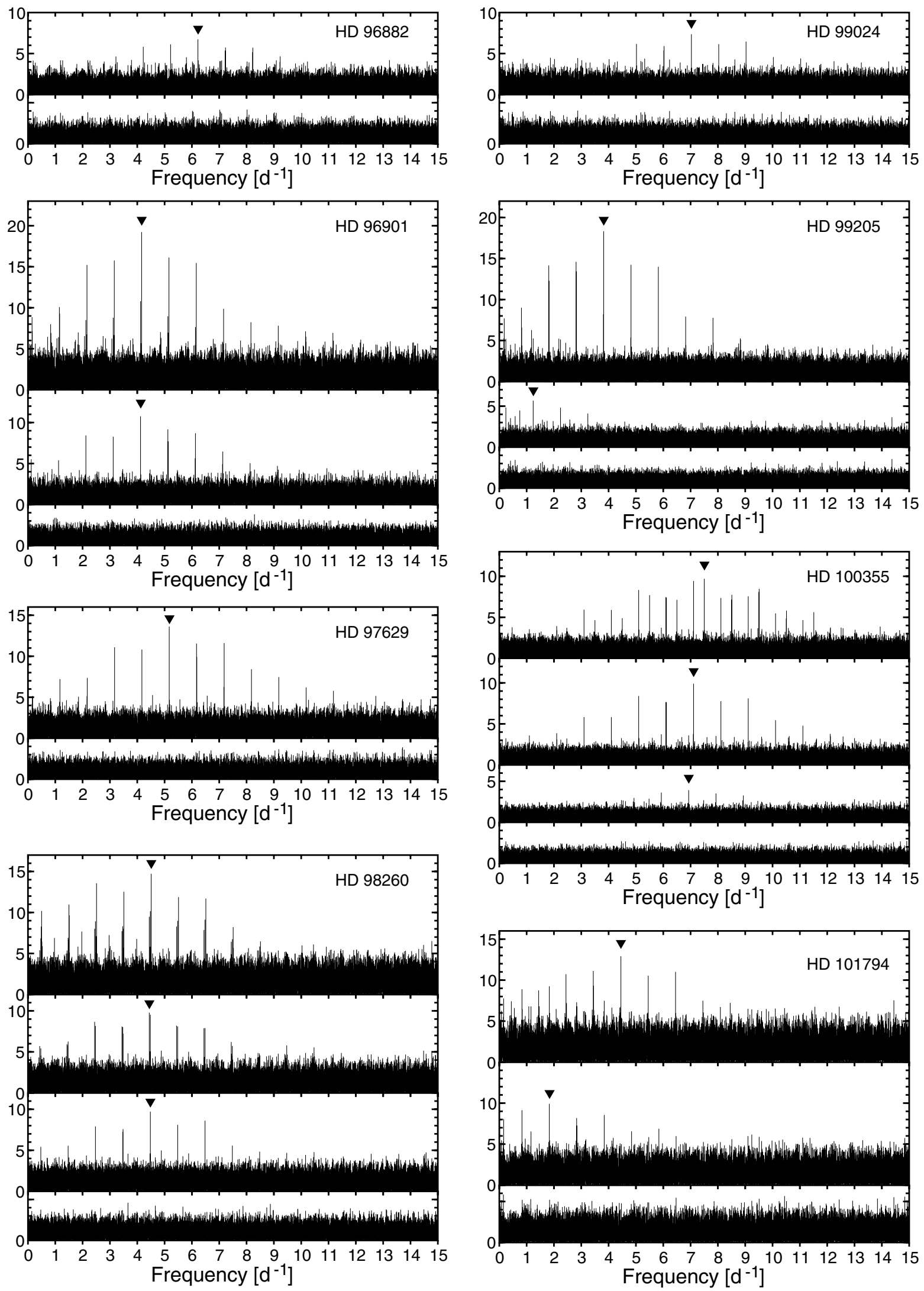

Fig. 5. The same as in Fig. 2, but for HD 96882, 96901, 97629, 98260, 99024, 99205, 100355, and 101794. 
A. Pigulski and G. Pojmański: $\beta$ Cephei stars in the ASAS-3 data. II., Online Material $p 10$
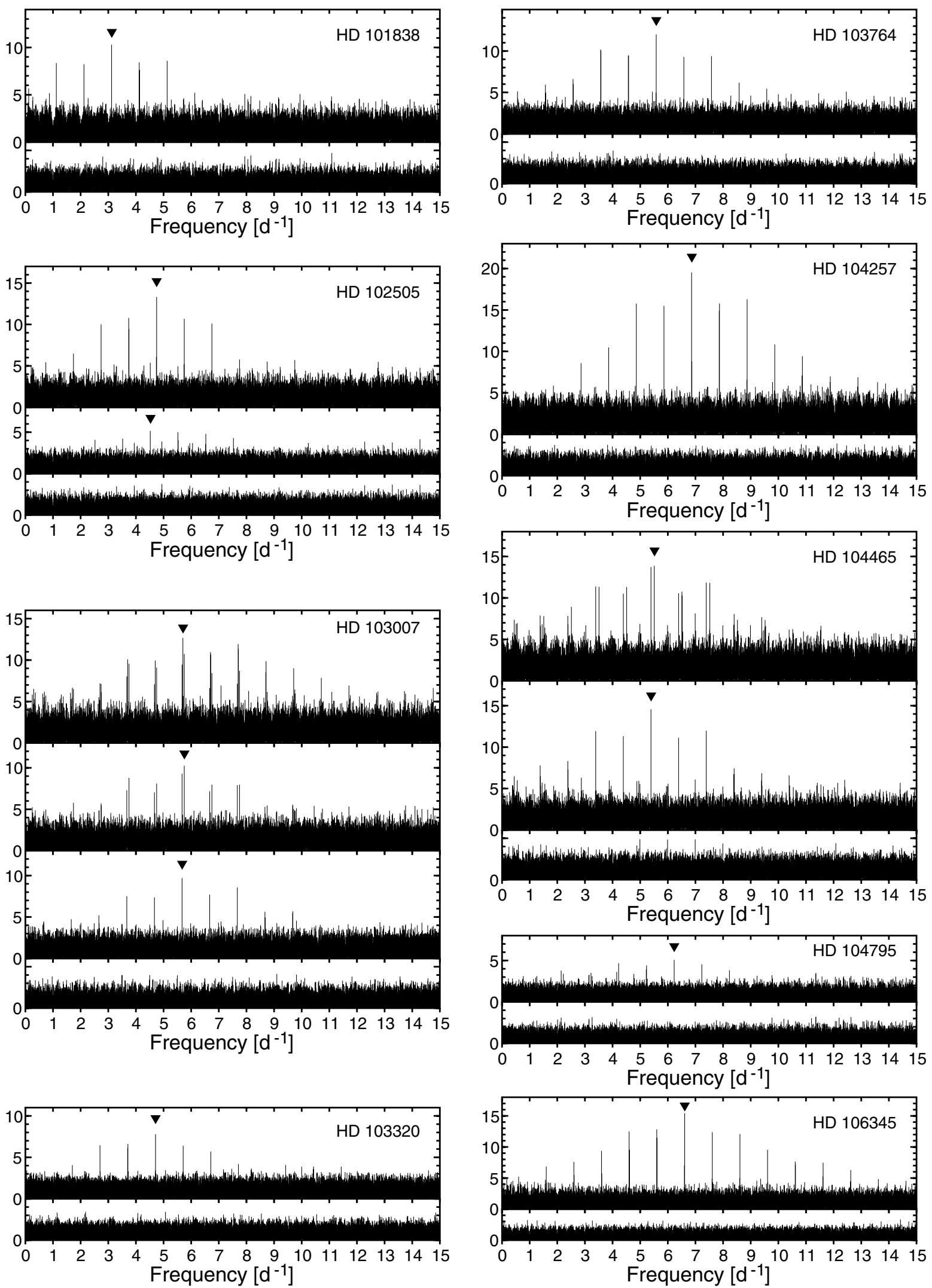

Fig. 6. The same as in Fig. 2, but for HD 101838, 102505, 103007, 103320, 103764, 104257, 104465, 104795, and 106345. 
A. Pigulski and G. Pojmański: $\beta$ Cephei stars in the ASAS-3 data. II., Online Material $p 11$
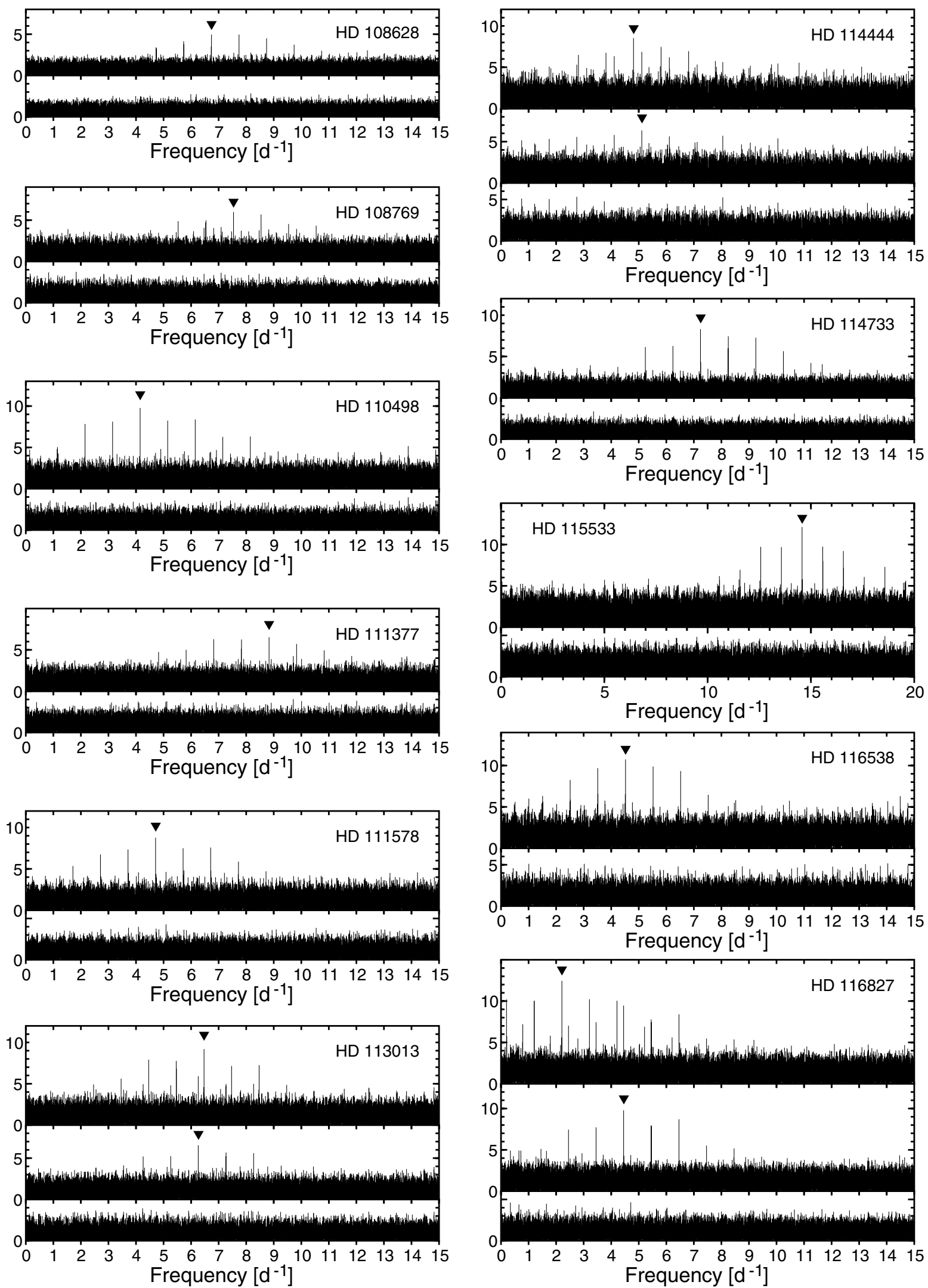

Fig. 7. The same as in Fig. 2, but for HD 108628, 108769, 110498, 111377, 111578, 113013, 114444, 114733, 115533,116538 , and 116827. 
A. Pigulski and G. Pojmański: $\beta$ Cephei stars in the ASAS-3 data. II., Online Material $p 12$
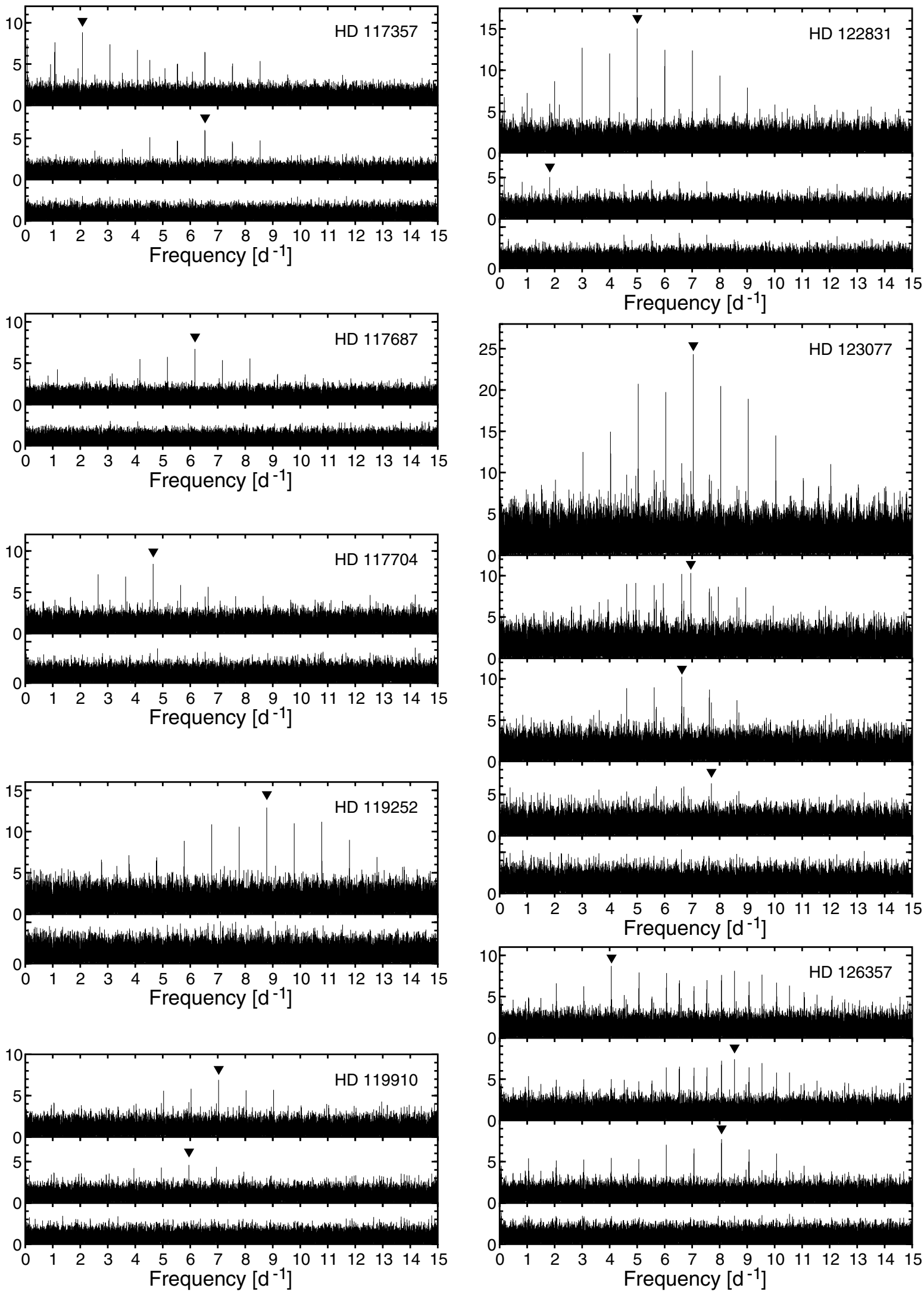

Fig. 8. The same as in Fig. 2, but for HD 117357, 117687, 117704, 119252, 119910, 122831, 123077, and 126357. 
A. Pigulski and G. Pojmański: $\beta$ Cephei stars in the ASAS-3 data. II., Online Material $p 13$
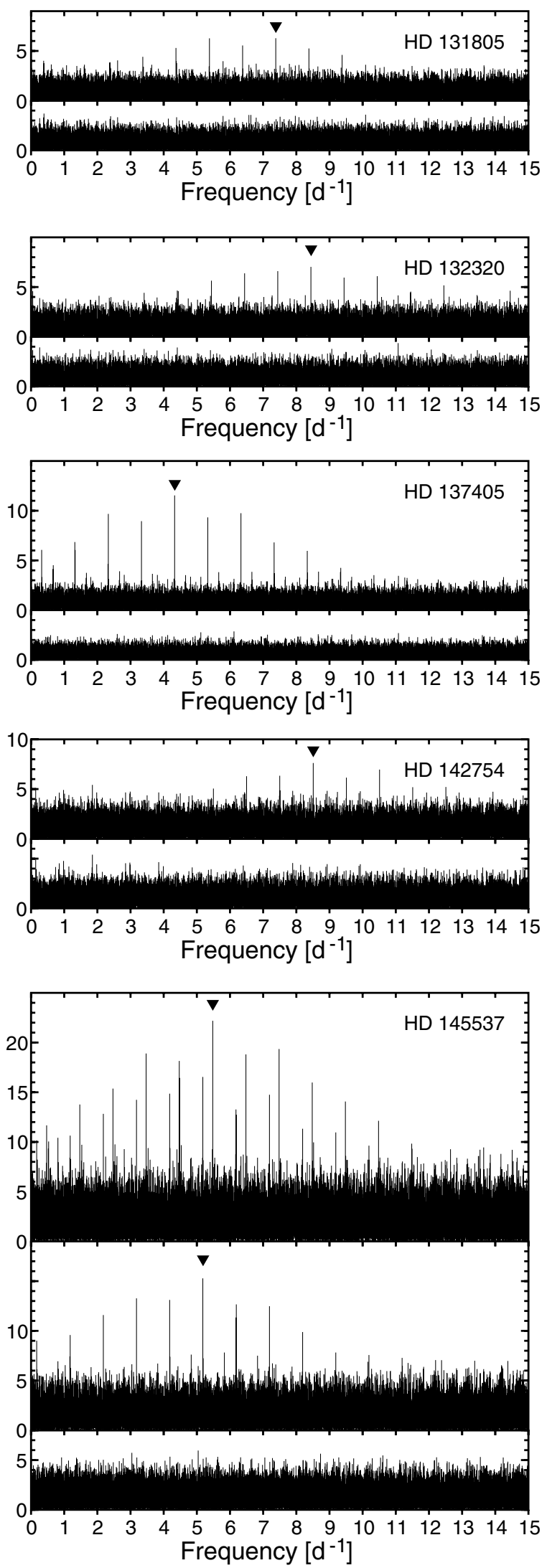
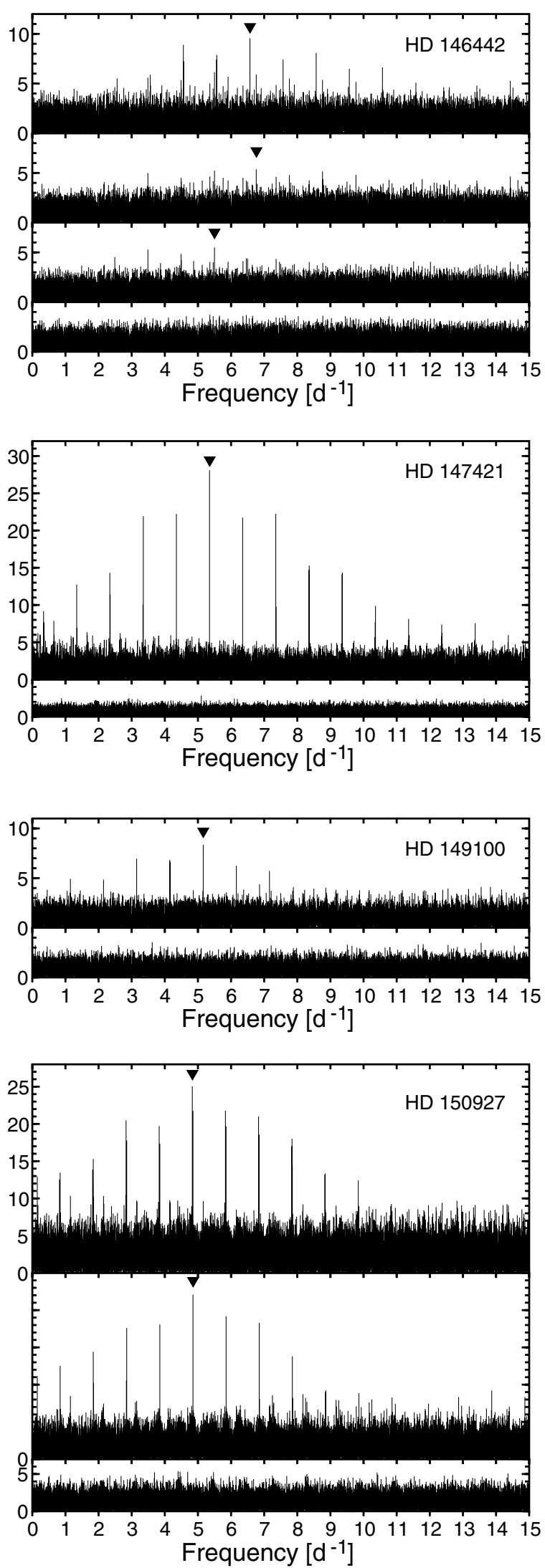

Fig. 9. The same as in Fig. 2, but for HD 131805, 132320, 137405, 142754, 145537, 146442, 147421, 149100, and 150927. 
A. Pigulski and G. Pojmański: $\beta$ Cephei stars in the ASAS-3 data. II., Online Material $p 14$
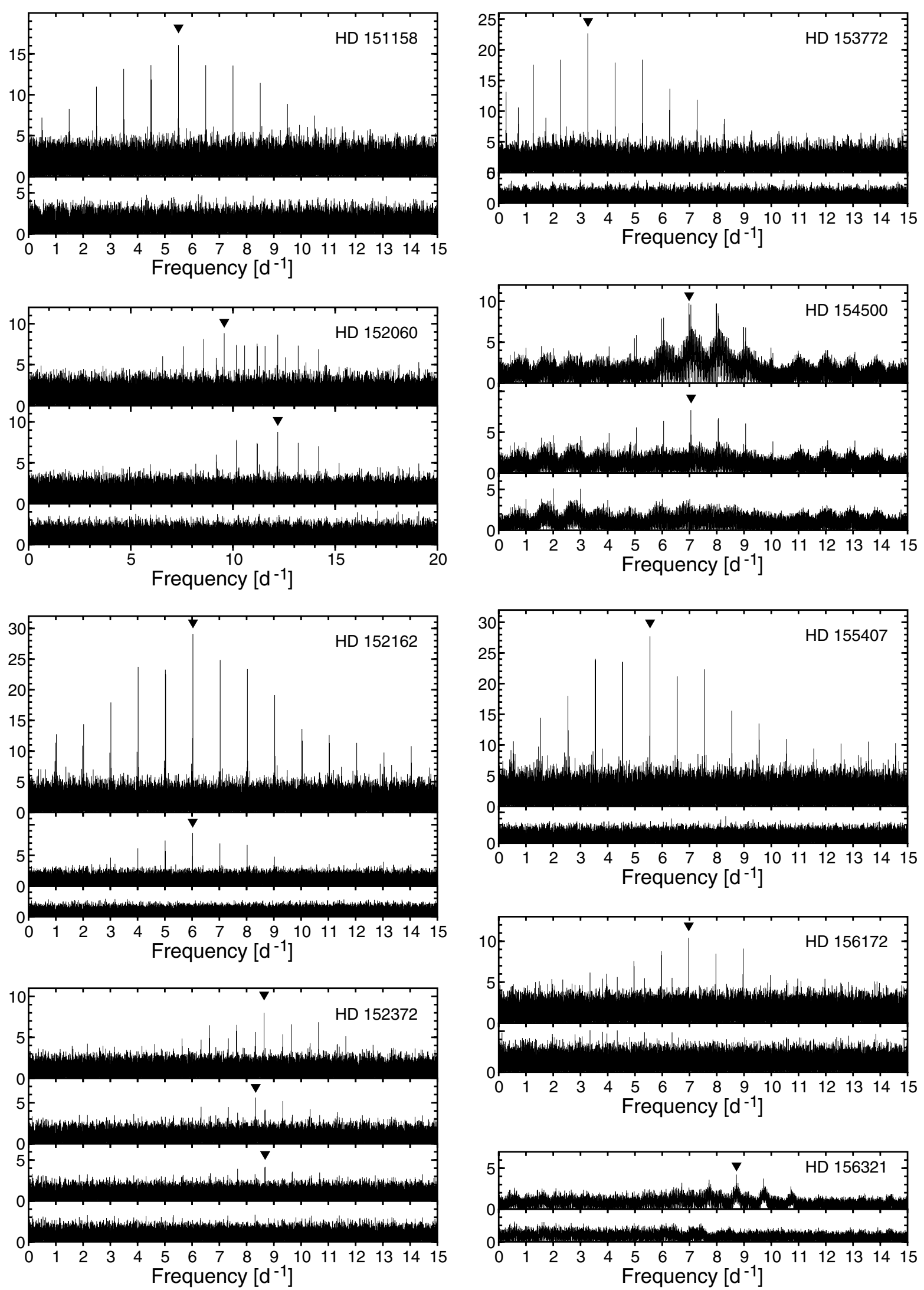

Fig. 10. The same as in Fig. 2, but for HD 151158, 152060, 152162, 152372, 153772, 154500, 155407, 156172, and 156321. 
A. Pigulski and G. Pojmański: $\beta$ Cephei stars in the ASAS-3 data. II., Online Material $p 15$
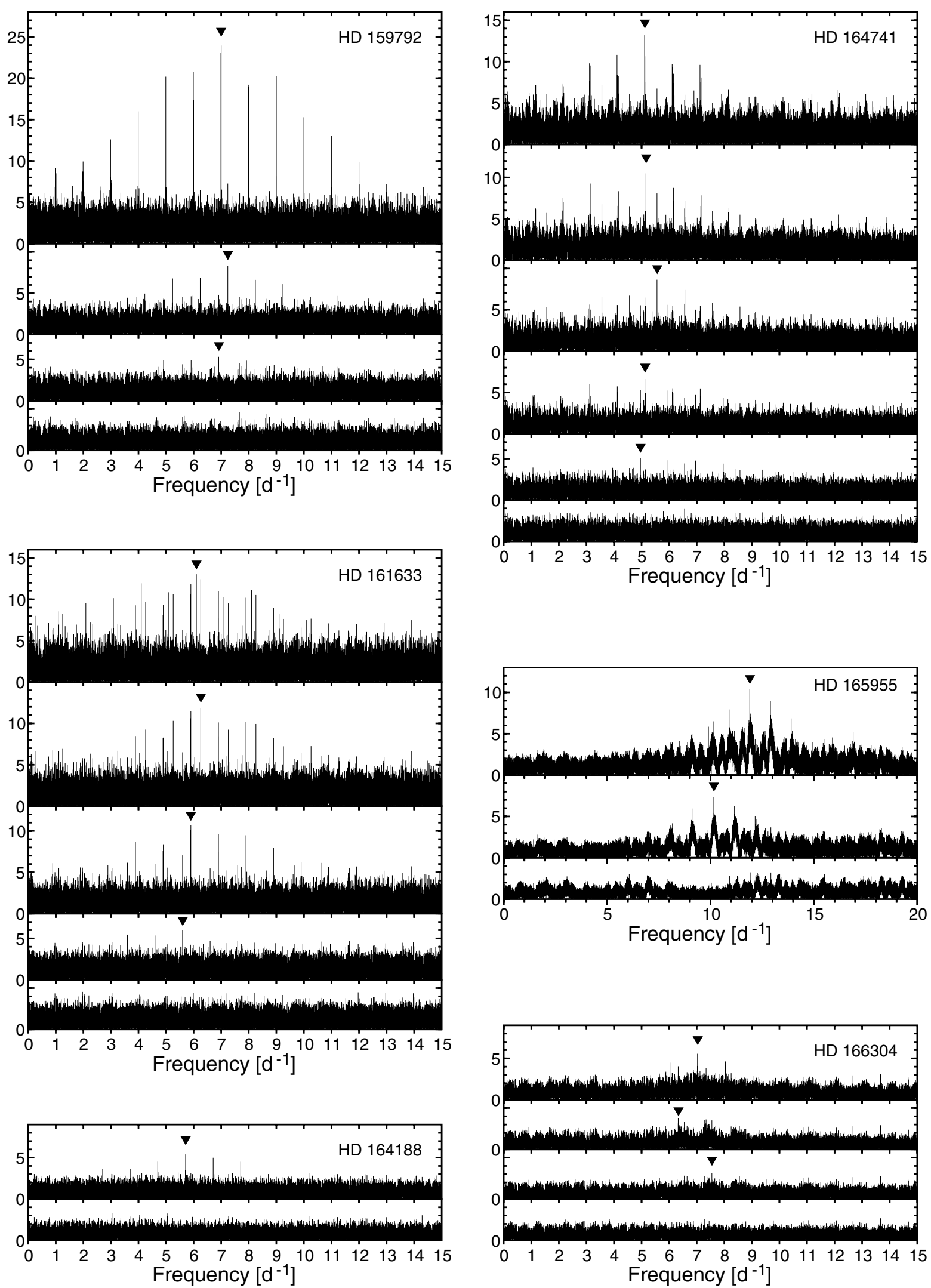

Fig. 11. The same as in Fig. 2, but for HD 159792, 161633, 164188, 164741, 165955, and 166304. 
A. Pigulski and G. Pojmański: $\beta$ Cephei stars in the ASAS-3 data. II., Online Material $p 16$
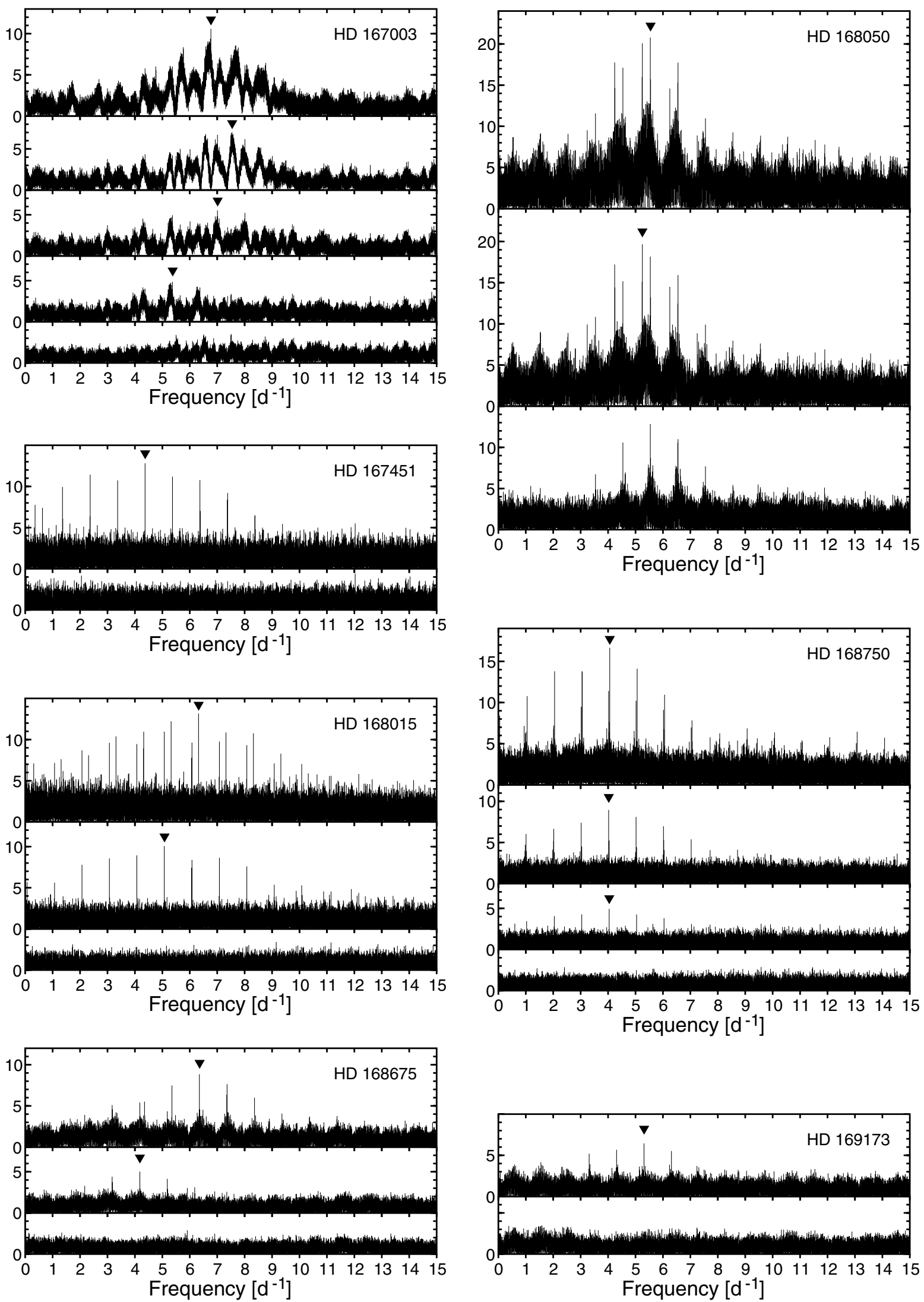

Fig. 12. The same as in Fig. 2, but for HD 167003, 167451, 168015, 168050, 168675, 168750, and 169173. 
A. Pigulski and G. Pojmański: $\beta$ Cephei stars in the ASAS-3 data. II., Online Material $p 17$
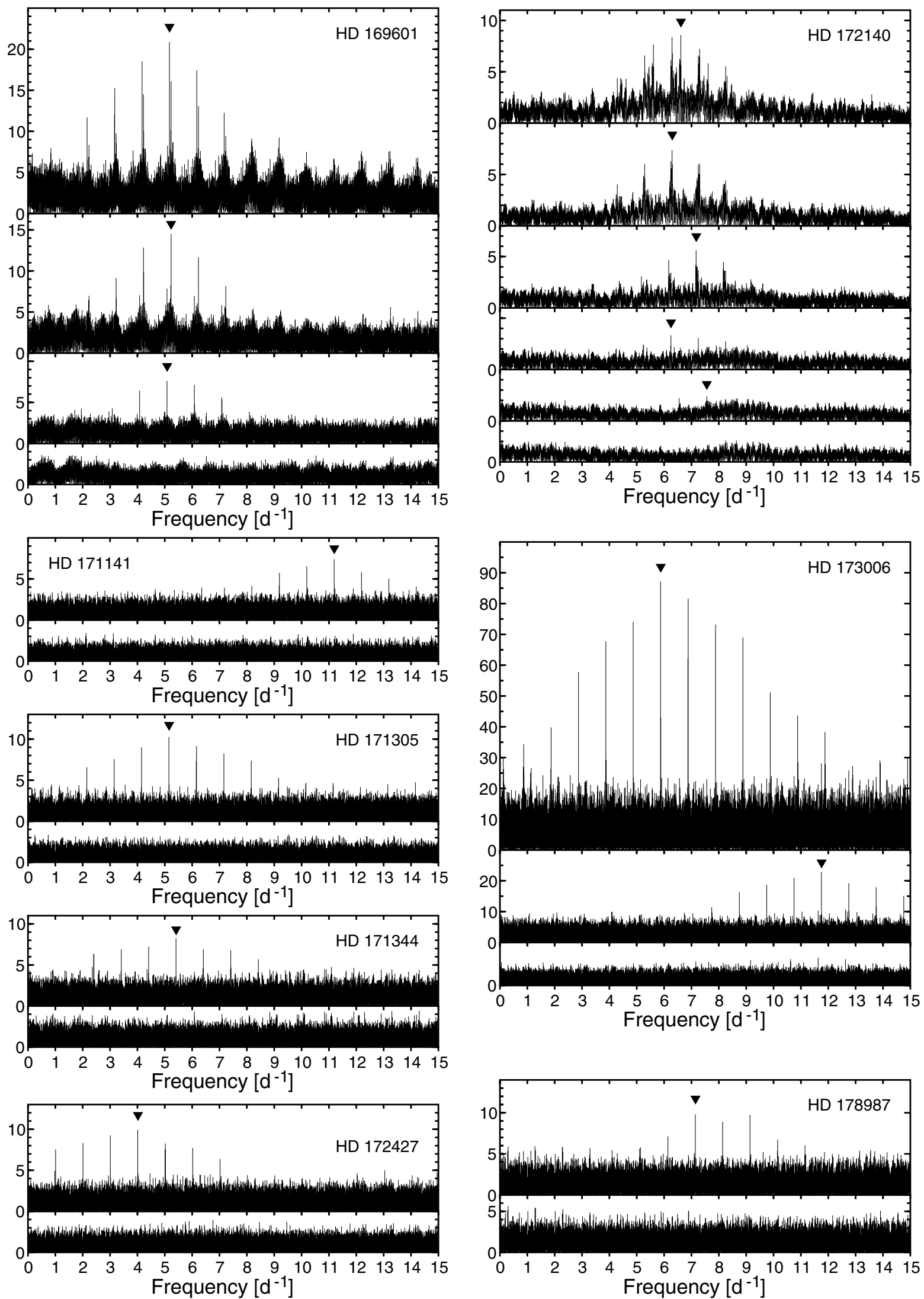

Fig. 13. The same as in Fig. 2, but for HD 169601, 171141, 171305, 171344, 172140, 172427, 173006, and 178987. 
A. Pigulski and G. Pojmański: $\beta$ Cephei stars in the ASAS-3 data. II., Online Material $p 18$
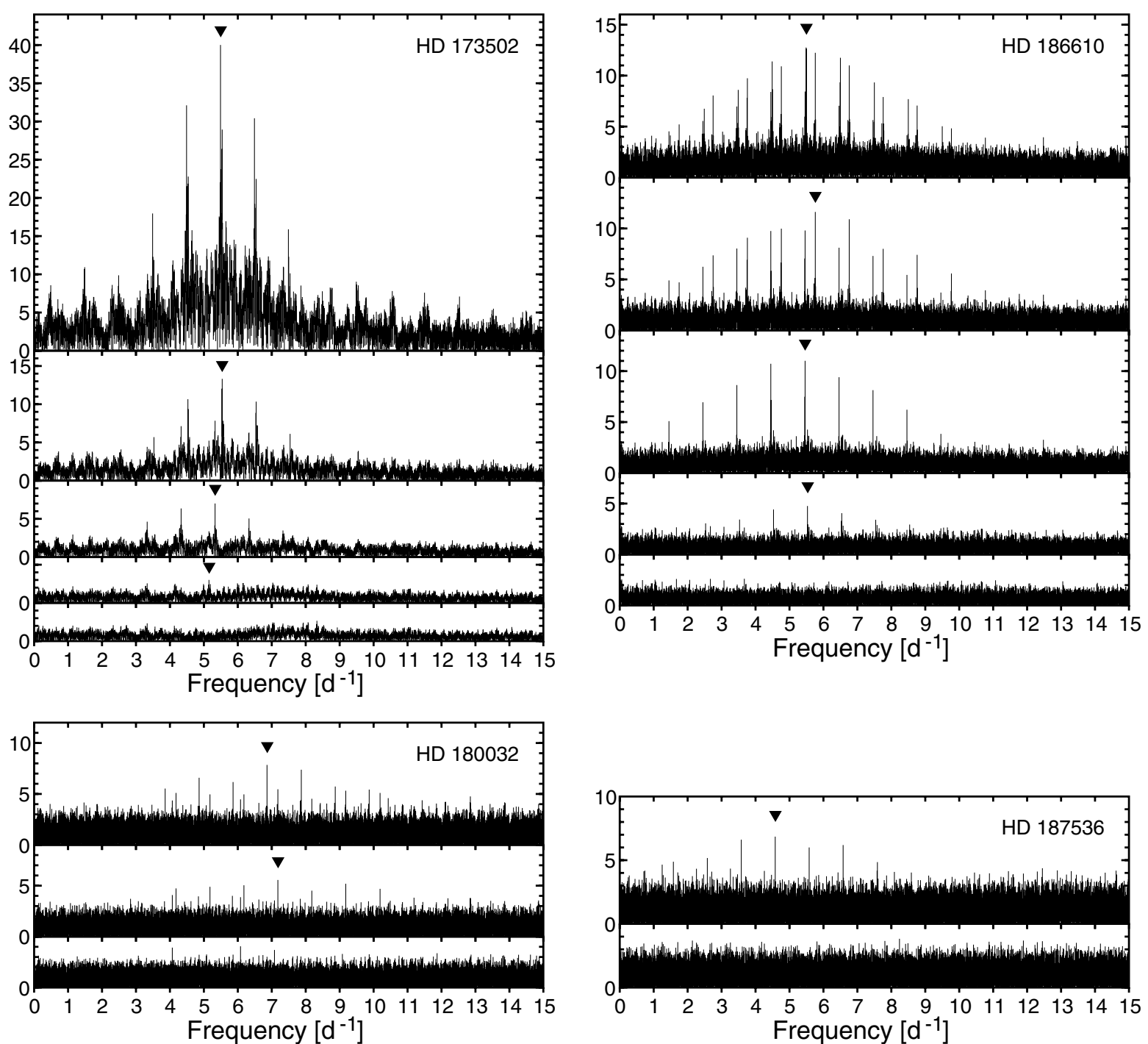

Fig. 14. The same as in Fig. 2, but for HD 173502, 180032, 186610, and 187536. 